\title{
Fundamental solutions for the wave operator on static Lorentzian manifolds with timelike boundary
}

\author{
Claudio Dappiaggi ${ }^{1,2, a}$, Nicoló Drago ${ }^{1,2, b}$, Hugo Ferreira ${ }^{1,3, c}$ \\ ${ }^{1}$ Istituto Nazionale di Alta Matematica \\ Sezione di Pavia - Via Ferrata, 5, 27100 Pavia, Italy. \\ 2 Dipartimento di Fisica \\ Università di Pavia - Via Bassi 6, 27100 Pavia, Italy. \\ ${ }^{3}$ INFN, Sezione di Pavia - Via Bassi 6, 27100 Pavia, Italy. \\ ${ }^{a}$ claudio.dappiaggi@unipv.it, ${ }^{b}$ nicolo.drago@unipv.it , ${ }^{c}$ hugo.ferreira@pv.infn.it
}

April 12, 2019

\begin{abstract}
We consider the wave operator on static, Lorentzian manifolds with timelike boundary and we discuss the existence of advanced and retarded fundamental solutions in terms of boundary conditions. By means of spectral calculus we prove that answering this question is equivalent to studying the selfadjoint extensions of an associated elliptic operator on a Riemannian manifold with boundary $(M, g)$. The latter is diffeomorphic to any, constant time hypersurface of the underlying background. In turn, assuming that $(M, g)$ is of bounded geometry, this problem can be tackled within the framework of boundary triples. These consist of the assignment of two surjective, trace operators from the domain of the adjoint of the elliptic operator onto an auxiliary Hilbert space $\mathrm{h}$, which is the third datum of the triple. Self-adjoint extensions of the underlying elliptic operator are in one-to-one correspondence with self-adjoint operators $\Theta$ on h. On the one hand, we show that, for a natural choice of boundary triple, each $\Theta$ can be interpreted as the assignment of a boundary condition for the original wave operator. On the other hand, we prove that, for each such $\Theta$, there exists a unique advanced and retarded fundamental solution. In addition, we prove that these share the same structural property of the counterparts associated to the wave operator on a globally hyperbolic spacetime.
\end{abstract}

\section{Introduction}

The existence and the characterization of the fundamental solutions of the D'Alembert wave operator $\square$ on a Lorentzian manifold $(N, g)$ is a classical problem which has been thoroughly studied in many contexts. Particularly if the underlying background is globally hyperbolic, a complete answer is known, cf. [BGP07, showing that there exist two unique distributions $\mathcal{G}^{ \pm} \in \mathcal{D}^{\prime}(N \times N)$, called advanced $(-)$ and retarded (+) fundamental solutions whose associated Green operators $\mathrm{G}^{ \pm}: \mathcal{D}(N) \rightarrow C^{\infty}(N)$ are such that $\square \circ \mathrm{G}^{ \pm}=\mathrm{G}^{ \pm} \circ \square=\left.\mathrm{id}\right|_{\mathcal{D}(N)}$ and, for any $f \in \mathcal{D}(N)$, $\operatorname{supp}\left(\mathrm{G}^{ \pm}(f)\right) \subseteq J^{\mp}(\operatorname{supp}(f)), J^{ \pm}$being the causal future $(+)$ and past $(-)$. 
Completely different is the situation if $(N, g)$ is a Lorentzian manifold with timelike boundary $\left(\partial N, \iota_{N}^{*} g\right)$, where $\iota_{N}: \partial N \hookrightarrow N$ and where $\left(\partial N, \iota_{N}^{*} g\right)$ is a Lorentzian submanifold. In this case a complete, cohesive analysis of the existence of fundamental solutions is not available. Yet, this class of backgrounds contains several notable examples, such as anti-de Sitter (AdS) or asymptotically AdS spacetimes which play a key rôle in several models that have recently attracted a lot of attention for the study of the properties of the wave or of the Klein-Gordon equation, see for example [Bac12, Hol12, Wro17, Vas12.

In addition, fundamental solutions play a pivotal rôle in the covariant quantization of free field theories, most notably in the construction of the $*$-algebra of observables. As a matter of facts, focusing on Bosonic fields, the canonical commutation relations are implemented in terms of a $*$-ideal which, in turn, is built out of a symplectic form defined out of the fundamental solutions, so to encode the information of dynamics and causality, see e.g. BDH13, BD15. From this viewpoint, the question that we shall answer in this paper has a direct impact in our understanding of several physical systems, as existence of fundamental solutions guarantees on the one hand that any underlying quantum field theory can be constructed following the standard rationale. On the other hand, if these solutions turn out not to be unique, it will entail that each of the possible choices corresponds to a different physical quantum system, as it has been already observed in different contexts, e.g. [BDS18, DF17, DNP16].

More precisely, our last statement stems from the observation that, in comparison to the same class of linear hyperbolic, partial differential equations on globally hyperbolic backgrounds, the main structural difference in our setting lies in the fact that initial data are not sufficient to identify a unique solution, which can be obtained only if one supplements with a suitable boundary condition. From the perspective of fundamental solutions, this statement amounts to saying that one cannot expect unique, advanced and retarded Green's operators, rather their existence should be tied to the specific boundary conditions assigned on $\partial N$. The goal of this paper is to start a full-fledged analysis of this problem on a large class of manifolds with non-empty boundary. Observe that, although we restrict the attention to the scalar wave equation, a similar analysis could be done for vector valued partial differential equations. In the special case of the Dirac field a recent work, connected to this problem, has appeared in GM18.

We focus on globally hyperbolic, oriented and time-oriented Lorentzian manifolds $(N, h)$ with timelike boundary AFS18, such that there exists, in addition, an isometry between $(N, h)$ and a standard static spacetime $M \times_{\beta} \mathbb{R}$. This is a warped product between $\mathbb{R}$ and a Riemannian manifold $(M, g)$ with a non-empty boundary $\left(\partial M, \iota_{M}^{*} g\right)$ where $\iota_{M}: \partial M \hookrightarrow M$. Thereon, we consider the D'Alembert wave operator $\widetilde{\square}$ as well as the auxiliary problem $\square=-\partial_{t}^{2}+A, t$ being the coordinate along $\mathbb{R}$, while $A$ is a second-order, elliptic, partial differential operator built out of $g$ and of $\beta$. In this framework the advanced and retarded fundamental fundamental solutions are defined as $\mathcal{G}^{ \pm} \in \mathcal{D}^{\prime}(\stackrel{\circ}{N} \times \stackrel{\circ}{N}), \stackrel{\circ}{ } \doteq N \backslash \partial N$, whose associated Green operators $\mathrm{G}^{ \pm}: \mathcal{D}(\stackrel{\circ}{N}) \rightarrow \mathcal{D}^{\prime}(\stackrel{\circ}{N})^{\prime}$ satisfy $\square \circ \mathrm{G}^{ \pm}=\mathrm{G}^{ \pm} \circ \square=\left.\mathrm{id}\right|_{\mathcal{D}(\stackrel{\circ}{N})}$, while also enjoying the support property $\operatorname{supp}\left(\mathrm{G}^{ \pm}(f)\right) \subseteq J^{ \pm}(\operatorname{supp}(f))$, for all $f \in \mathcal{D}(N)$. On account of the underlying metric being static, as a first step one can reduce the problem to an auxiliary one obtained via the ansatz $\mathcal{G}^{+}=\theta\left(t-t^{\prime}\right) \mathcal{G}$ and $\mathcal{G}^{-}=-\theta\left(t^{\prime}-t\right) \mathcal{G}$, where $\theta$ is the Heaviside distribution while $\mathcal{G} \in \mathcal{D}^{\prime}(\stackrel{\circ}{N} \times \stackrel{\circ}{N})$ is a solution of

$$
\left\{\begin{array}{l}
(\square \otimes \mathbb{I}) \mathcal{G}=(\mathbb{I} \otimes \square) \mathcal{G}=0 \\
\left.\mathcal{G}\right|_{t=t^{\prime}}=0,\left.\quad \partial_{t} \mathcal{G}\right|_{t=t^{\prime}}=-\left.\partial_{t^{\prime}} \mathcal{G}\right|_{t=t^{\prime}}=\delta_{\operatorname{diag}(\stackrel{\circ}{M})},
\end{array},\right.
$$

where $\left.\right|_{t=t^{\prime}}$ indicates the pull-back of a distribution on $\stackrel{\circ}{N} \times \stackrel{\circ}{N}$ to $(\{t\} \times \stackrel{\circ}{M}) \times(\{t\} \times \stackrel{\circ}{M})$ while $\delta_{\text {diag }(\stackrel{M}{M})}$ is the Dirac delta distribution on the diagonal of $M \times \stackrel{\circ}{M}$. As it stands, the above initial value problem is incomplete unless one also assigns a boundary condition on $\partial N$ which allows for the identification of a unique solution. In order to understand which class of such conditions is admissible we focus our attention on $A$, which, being the metric static, is a partial differential operator acting on scalar functions over $M$. Our strategy proceeds in two steps. In the first we start by reading $A$ as a symmetric operator 
on $\mathrm{H} \equiv L^{2}\left(M ; \mathrm{d} \mu_{g}\right), \mathrm{d} \mu_{g}$ being the metric-induced volume form and we show that, for every self-adjoint extension of $A$, by means of spectral calculus, it is possible to construct $\mathcal{G}$, solution of the above system of partial differential equations.

In the second step instead we start by investigating which are the possible self-adjoint extensions of $A$ and how to characterize them explicitly in terms of boundary conditions. To this avail we require an additional assumption, namely $(M, g)$ ought to be of bounded geometry, a widely used structure which has been recently studied in the context of manifolds with boundary in AGN16, GS13, Sch01. On the one hand, we observe that our assumptions include several interesting scenarios such as anti-de Sitter spacetime. On the other hand, Riemannian manifolds with boundary and of bounded geometry turn out to be the natural framework to construct Sobolev spaces and to prove thereon a generalization of the standard Lions-Magenes trace theorems on $\mathbb{R}^{n}$. This last feature is of special interest to us since it allows us to classify and to characterize the self-adjoint extensions using the framework of boundary triples, Gru68] and [HSF12, Ch. 6].

In short and adapting it to the case at hand, the latter includes and even supersedes Von Neumann theory of deficiency indexes - see Pos18 for an equivalent approach based on resolvents. It associates to the symmetric operator $A$ an auxiliary Hilbert space, in our case chosen as $\mathrm{h} \equiv L^{2}\left(\partial M, \mathrm{~d} \mu_{\iota_{M}^{*} g}\right)$, together with two linear, surjective maps $\gamma_{i}: D\left(A^{*}\right) \rightarrow \mathrm{h}, i=0,1$ where $A^{*}$ is the adjoint of $A$, so that a Green's formula holds true:

$$
\left(A^{*} f \mid f^{\prime}\right)_{\mathrm{H}}-\left(f \mid A^{*} f^{\prime}\right)_{\mathrm{H}}=\left(\gamma_{1} f \mid \gamma_{0} f^{\prime}\right)_{\mathrm{h}}-\left(\gamma_{0} f \mid \gamma_{1} f^{\prime}\right)_{\mathrm{h}} \quad \forall f, f^{\prime} \in D\left(A^{*}\right) .
$$

In the case we are interested in, it turns out that $\gamma_{0}, \gamma_{1}$ can be built working at the level of Sobolev spaces over $M$ as combination between the above mentioned trace theorems and the normal derivative to $\partial M$. As a by-product, one can apply a standard result from the theory of boundary triples according to which there is a one-to-one correspondence between the self-adjoint operators $\Theta$ on $\mathrm{h}$ and the self-adjoint extensions of $A$, denoted by $A_{\Theta}$.

By using these results and the spectral calculus for $A_{\Theta}$ we are able to construct explicitly, for each choice of $\Theta$, fundamental solutions $\mathcal{G}_{\Theta}^{ \pm}$associated to $\square$, proving in addition with an energy estimate that they enjoy the sought support properties. In addition, we show that, for every $f \in \mathcal{D}(N)$, it holds that $\gamma_{1}\left(\mathrm{G}_{\Theta}^{ \pm}(f)\right)=\Theta \gamma_{0}\left(\mathrm{G}_{\Theta}^{ \pm}(f)\right)$, where $\mathrm{G}_{\Theta}^{ \pm}$are the advanced and retarded Green's operators associated with $\mathcal{G}_{\Theta}^{ \pm}$. This justifies our statement according to which fixing $\Theta$, and thus a pair of fundamental solutions $\mathcal{G}_{\Theta}^{ \pm}$, is subordinated to a choice of boundary condition. Furthermore, under mild assumptions on the warping factor $\beta$, so to ensure that the operator $A$ is uniformly elliptic, we are able to prove two additional results which strongly characterize the advanced and retarded Green's operators. On the one hand, we show that $\mathrm{G}_{\Theta}^{ \pm}[\mathcal{D}(N)] \subset C^{\infty}(N)$, on the other hand we are able to enlarge the domain of $\mathrm{G}_{\Theta} \doteq \mathrm{G}_{\Theta}^{-}-\mathrm{G}_{\Theta}^{+}$so to construct an exact sequence of linear maps, which characterize completely, for every admissible $\Theta$, the space of associated smooth solutions of the equation of motion ruled by $\square$.

Finally we observe that, although the class of boundary conditions that we define in terms of the selfadjoint extensions of $A$ is rather large including for example those of Robin type, it does not encompass some cases which are often discussed in the literature. Most notably it might be desirable to allow an explicit time-dependence of the boundary condition, as it happens for example in those of Wentzell type, which have remarkable applications in several models, see [Fel57, Ue73, Z18] and GGG03 in particular. The last part of this work is devoted to an analysis of such scenario. We show that it is possible to extend the range of applicability of the framework of boundary triples proving in particular the existence of fundamental solutions for a larger class of boundary conditions, including those of Wentzell type.

The structure of the paper is the following: In Section 1.1 we introduce the basic geometric data of this paper. In particular, we emphasize the notion of a static Lorentzian spacetime with timelike 
boundary and we outline the notion of manifold with boundary and of bounded geometry, reviewing its main properties. In Section 1.2 we introduce Sobolev spaces on Riemannian manifolds of bounded geometry and we put a particular emphasis on the trace theorem as proven in GS13. In Section 2 we discuss the framework of boundary triples, first from an abstract point of view and then we specialize it to the case of a second-order, elliptic partial differential operators, making a direct connection to the theory of Sobolev spaces outlined in the previous section. In particular, we use boundary triples to characterize the self-adjoint extensions of symmetric operators with equal deficiency indexes. In Section 3 we obtain the main results of this work. Here we start from the D'Alembert wave operator on a static Lorentzian spacetime and we rewrite it in terms of an equivalent operator of the form $\square=-\partial_{t}^{2}+A$, where $A$ is a second-order, elliptic partial differential operator, symmetric on $L^{2}\left(M ; \mathrm{d} \mu_{g}\right)$. As a first step, by using spectral analysis, we prove the existence of advanced and retarded Green's operators for any choice of self-adjoint extension of $A$. Via a suitable boundary triple, these are in turn put into a one-to-one relation with the choice of a self-adjoint operator on $L^{2}\left(\partial M ; \mathrm{d} \mu_{i_{M}^{*}} g\right)$. Subsequently, we prove several structural properties of the fundamental solutions, ranging from the support to the characterization of the relation between the choice of boundary condition for $\square$ and the self-adjoint extension of $A$. Furthermore, under additional mild conditions on the metric, we show that all smooth solutions to the wave equation can be written in terms of the advanced and retarded Green's operators which thus turn out to encompass as much information on the underlying operator ruling the dynamics as their natural counterpart on globally hyperbolic spacetimes. At last, in Section 3.1, we extend the previous framework to account also for a larger class of time-dependent boundary condition, including in particular those of Wentzell type.

\subsection{Geometric data}

The goal of this section is to introduce both the geometric data and the key functional spaces at the heart of this work, fixing in particular the notations and conventions. With respect to the structure of Lorentzian manifolds with empty boundary we refer mainly to BEE96, while, for the case with a non-empty, timelike boundary, recent analyses are available in CGS09, Sol06. It is noteworthy that our framework can be read as a special instance of that considered and studied in AFS18.

Following the standard definition, see for example [Lee00, Ch. 1], in this paper both the symbols $M$ and $N$ refer to a smooth, second-countable, connected, manifold of dimension $m \geq 1$ (resp. $m+1)$, with smooth boundary $\partial M($ resp. $\partial N)$. A point $p \in M($ resp. $N)$ such that there exists an open neighbourhood $U$ containing $p$, diffeomorphic to an open subset of $\mathbb{R}^{m}$ (resp. $\left.\mathbb{R}^{m+1}\right)$, is called an interior point and the collection of these points is indicated with $\operatorname{Int}(M) \equiv \stackrel{\circ}{M}(\operatorname{resp} . \quad \operatorname{Int}(N) \equiv \stackrel{N}{N})$. As a consequence $\partial M($ resp. $\partial N)$, if non empty, can be read as a manifold on its own and $\partial M=M \backslash M($ resp . $\partial N=N \backslash \stackrel{\circ}{N})$.

Definition 1: We say that $N$ is a Lorentzian manifold with timelike boundary if it is oriented, time oriented and endowed with a smooth Lorentzian metric $h$ such that also $\iota_{N}^{*} h$ is a Lorentzian metric, $\iota_{N}$ being the embedding of $\partial N$ in $N$.

In the class of Lorentzian manifolds with timelike boundary $(N, h)$, we will be interested in those which are standard static, that is for which there exists a nowhere vanishing, irrotational, timelike Killing field $\chi \in \Gamma(T N)$, cf. [BEE96, Lemma 3.78] and [San06], and $(N, h)$ is isometric to the warped product $M \times_{\beta} \mathbb{R}$, with line element

$$
h=-\beta \mathrm{d} t^{2}+g,
$$

where $t: M \times \beta \mathbb{R} \rightarrow \mathbb{R}$ is the projection along the second component, playing thus the role of time variable, $\beta \in C^{\infty}(M ;(0, \infty))$ and $g$ identifies a time-independent Riemannian metric on each submanifold $\{t\} \times M$. As a consequence 
Corollary 2: Let $(N, h)$ be a standard static, Lorentzian manifold with timelike boundary. Then also $\partial N$ is a standard static Lorentzian manifold.

Proof. Per hypothesis $(N, h)$ is isometric to $M \times_{\beta} \mathbb{R}$ with line element (1) where $\partial_{t}$ plays the role of the complete, irrotational, timelike, nowhere vanishing Killing field. Hence $\partial N$ is isometric to $\partial M \times_{\beta} \mathbb{R}$ and, calling $\iota_{M}: \partial M \rightarrow M$ the embedding map, (1) reduces to $-\widetilde{\beta} \mathrm{d} t^{2}+\iota_{M}^{*}(g),\left.\widetilde{\beta} \doteq \beta\right|_{\partial M}$. As a consequence $\partial N$ has the sought property.

Observe that, with these data, $M$ comes equipped with an induced orientation and with a smooth Riemannian metric $g$, so that $(\partial M, \widetilde{g})$ is also an oriented Riemannian manifold if endowed with the induced orientation and metric, that is $\widetilde{g} \doteq \iota_{M}^{*}(g), \iota_{M}$ being the embedding of $\partial M$ in $M$. In the class of Riemannian manifolds with non-empty boundary, we are interested in a particular subclass, distinguished by its geometric properties in a neighbourhood of $\partial M$. The following definitions were first given in AGN16, GN17, barring the next one, which is standard, $c f$. Eich91]:

Definition 3: A Riemannian manifold $(M, g)$ with $\partial M=\emptyset$ is called of bounded geometry if the injectivity radius $r_{\text {inj }}(M)>0$ and if $T M$ is of totally bounded curvature, that is $\left\|\nabla^{k} R\right\|_{L^{\infty}(M)}<\infty$ for all $k \in \mathbb{N} \cup\{0\}, R$ being the scalar curvature and $\nabla$ the Levi-Civita connection associated with $g$.

To avoid the problem that $r_{\mathrm{inj}}(M)$ vanishes whenever $\partial M \neq \emptyset$, one must first consider the following generalization:

Definition 4: Let $(M, g)$ be a Riemannian manifold of bounded geometry and let $\left(Y, \iota_{Y}\right)$ be a codimension $k$ closed, embedded, smooth submanifold with an inward pointing, unit normal vector field $\nu_{Y}$. We say that $\left(Y, \iota_{Y}^{*} g\right)$ is a bounded geometry submanifold if the following holds:

1. the second fundamental form $K_{Y}$ of $Y$ in $M$ together with all its covariant derivatives along $Y$ is bounded,

2. there exists $\epsilon_{Y}>0$ such that the map $\varphi_{\nu_{Y}}: Y \times\left(-\epsilon_{Y}, \epsilon_{Y}\right) \rightarrow M$ defined as $(x, z) \mapsto \varphi_{\nu_{Y}}(x, z) \doteq$ $\exp _{x}\left(z \nu_{Y, x}\right)$ is injective, where $\exp _{x}$ is the exponential map of $M$ at $x$.

We observe that, as proven in AGN16, Definition \entails that $\left(Y, \iota_{Y}^{*} g\right)$ is automatically a Riemannian manifold of bounded geometry. We introduce the class of Riemannian manifolds we will be working with in this paper:

Definition 5: Let $(M, g)$ be a Riemmannian manifold with non-empty boundary $\partial M$. We say that $(M, g)$ has bounded geometry if there exists a Riemannian manifold of bounded geometry $(\widehat{M}, \widehat{g})$ of the same dimension of $M$ such that

1. $M \subset \widehat{M}$ and $g=\left.\widehat{g}\right|_{M}$,

2. $\left(\partial M, \iota^{*} \widehat{g}\right)$ is a bounded geometry submanifold of $\widehat{M}$, where $\iota: \partial M \rightarrow \widehat{M}$ is the embedding man? 1 .

Remark 6: Definition [5 is equivalent to the original one of manifolds with boundary and of bounded geometry given by Schick in Sch01. We observe that, while the definition given in this last cited paper does not require any extrinsic data such as in particular $\widehat{M}$, all results obtained are independent from the choice of the latter.

Since, from now on, we will be working only with Riemannian manifolds with non-empty boundary and of bounded geometry, we will drop the subscript $Y$ as in Definition 4 since we will be always referring

\footnotetext{
${ }^{1}$ Recall that, if we consider the restriction map res $: \widehat{M} \rightarrow M$, then res $\circ \iota=\iota$. Hence $\iota^{*} \widehat{g}=\iota^{*} g$.
} 
to $\partial M$ as the embedded submanifold. In addition we call geodesic collar (of $\partial M$ ) the set $\partial M \times[0, \epsilon$ ) such that the map $\varphi_{\nu}$ is a diffeomorphism onto its image and we define

$$
\mathcal{G C}_{\epsilon}(M) \doteq \varphi_{\nu}[\partial M \times[0, \epsilon)] .
$$

Remark 7: We observe that all Riemannian manifolds with compact boundary meet the requirements of Definition 5. At the same time one can also consider non-compact boundaries such as for example $\mathbb{H}^{n}$, the collection of all points $\left(x_{1}, \ldots, x_{n}\right) \in \overline{\mathbb{R}_{+}} \times \mathbb{R}^{n-1}$ endowed with the Euclidean metric of $\mathbb{R}^{n}$.

As a next step, if $(M, g)$ is a Riemannian manifold of bounded geometry, we can introduce a distinguished set of coordinates which are at the heart of the definition of Sobolev spaces and of the associated trace theorem proven in GS13. Here, we will recall only the basic structures and facts, leaving all details and proofs to [AGN16, Sec. 4.2] and to [GS13, Sec. 4.1]. Note that the following construction was used in Sch01 though with the name of normal collar coordinates.

Let $(M, g)$ be a Riemannian manifold with boundary and of bounded geometry as per Definition 5. For any $p \in \partial M$, we can choose any orthonormal basis of $T_{p} \partial M$ to identify it with $\mathbb{R}^{m-1}, m=\operatorname{dim} M$. From now on this identification will be left implicit. Since the injectivity radius of $\partial M$ is finite, for all $0<r<r_{\text {inj }}(\partial M)$, the exponential map $\exp _{p}^{\partial M}: \mathcal{B}^{m-1}(0) \rightarrow \mathcal{B}_{r}(p)$ is a diffeomorphism. Here $\mathcal{B}^{m-1}(0)$ stands for the ball of radius $r$ in $\mathbb{R}^{m-1}$ centered at 0 . By considering in addition the map $\varphi_{\nu}$ for $\partial M$ (see Definition 4 and (2) $)$, whenever $0<r<\min \left\{\frac{r_{\mathrm{inj}}(\partial M)}{2}, \frac{r_{\mathrm{inj}}(M)}{4}, \frac{\epsilon}{2}\right\}$, we identify the following:

$$
\left\{\begin{array}{lll}
\kappa_{p}: \mathcal{B}^{m-1}(0) \times[0, r) \rightarrow M, & \kappa_{p}(x, z) \doteq \varphi_{\nu}\left(\exp _{p}^{\partial M}(x), z\right), & p \in \partial M \\
\kappa_{p}: \mathcal{B}_{r}^{m}(0) \rightarrow M, & \kappa_{p}(v) \doteq \exp _{p}^{M}(v), & p \in \stackrel{\stackrel{M}{M}}{ }
\end{array}\right.
$$

where $\mathcal{B}_{r}^{m}(0)$ indicates the ball of radius $r$ centered at the origin of $T_{p} M$, here implicitly identified with $\mathbb{R}^{m}$. Let $U_{p}(r)$ stand for the image in $M$ of the map $\kappa_{p}$, then we can define the following:

Definition 8: Let $(M, g)$ be a Riemannian manifold with boundary and of bounded geometry and let $0<$ $r<\min \left\{\frac{r_{\mathrm{inj}}(\partial M)}{2}, \frac{r_{\mathrm{inj}}(M)}{4}, \frac{\epsilon}{2}\right\}$. We call Fermi (or normal collar) chart the map $\kappa_{p}: \mathcal{B}^{m-1}(0) \times[0, r) \rightarrow M$ for $p \in \partial M$. The ensuing coordinates $\left(x^{i}, z\right): U_{p}(r) \rightarrow \mathbb{R}^{m-1} \times[0, \infty), i=1, \ldots, m-1$ are called Fermi (or normal collar) coordinates.

If the point $p$ does not lie on the boundary of $M$, one can adapt straightforwardly this last definition to obtain the standard normal geodesic coordinates. Since we will not make use of them, we omit giving an explicit expression.

To conclude the section, we study the interplay between Riemannian manifolds with boundary and of bounded geometry and standard static, Lorentzian manifolds with a timelike boundary.

Proposition 9: Let $(M, g)$ be a Riemannian manifold with boundary and of bounded geometry and let $(\widehat{M}, \widehat{g})$ be a Riemannian manifold of bounded geometry such that $M \subset \widehat{M}$ and $g=\left.\widehat{g}\right|_{M}$. Then

1. Every $\beta \in C^{\infty}(M ;(0, \infty))$ identifies an isometry class $[(N, h)]$ of standard static, Lorentzian manifolds with timelike boundary,

2. If in addition there exists $\widehat{\beta} \in C^{\infty}(\widehat{M} ;(0, \infty))$ such that $\left.\widehat{\beta}\right|_{M}=\beta$ and if $\frac{\widehat{g}}{\beta}$ identifies a complete Riemannian metric on $\widehat{M}$, then each representative $(N, h)$ of $[(N, h)]$ is a submanifold of a standard static, globally hyperbolic spacetime $(\widehat{N}, \widehat{h})$.

A manifold $(N, h)$ satisfying condition 1 . of Proposition 9 will be called a static Lorentzian spacetime, with timelike boundary. 
Proof. Consider $M$ as per hypothesis and construct the warped product $M \times_{\beta} \mathbb{R}$ endowed with the line element $\mathrm{d} s^{2}=-\beta \mathrm{d} t^{2}+g$ where $t: M \times_{\beta} \mathbb{R} \rightarrow \mathbb{R}$ is the projection along the second component. Every manifold $(N, h)$ which is isometric to $M \times_{\beta} \mathbb{R}$ with the given metric is standard static, hence proving the first point. To prove the second statement, it suffices to observe that $M \times_{\beta} \mathbb{R}$ can be seen as being isometrically embedded in the standard static spacetime $\widehat{M} \times_{\widehat{\beta}} \mathbb{R}$ with line element $\mathrm{d} s^{2}=-\widehat{\beta} \mathrm{d} t^{2}+\widehat{g}$. This manifold is globally hyperbolic on account of [BEE96, Theorem 3.66].

Remark 10: Observe that condition 2 in the last proposition is a constraint only on the admissible functions $\widehat{\beta}$. As a matter of fact, every Riemannian manifold of bounded geometry is metric complete Eich91 and thus $\frac{\widehat{g}}{\hat{\beta}}$ is also complete if and only if $\widehat{\beta}$ behaves at most quadratically at infinity, cf. San06, Rem. 2.2].

\subsection{Sobolev spaces on manifolds of bounded geometry}

We shall introduce the functional spaces that we will be using in the next sections, as well as their main properties. We will be using most of the results from GS13. In the following we consider $(\widehat{M}, \widehat{g})$, a Riemannian manifold of bounded geometry such that $\partial \widehat{M}=\emptyset$. The case with non empty boundary has been discussed mainly in AGN16. With $\mathcal{D}(\widehat{M})$ we will indicate the space of smooth, compactly supported functions on $\widehat{M}$ endowed with the standard locally convex topology, while with $L^{p}(\widehat{M}) \doteq L^{p}\left(\widehat{M}, \mathrm{~d} \mu_{\widehat{g}}\right)$, $p \in \mathbb{N}$ we consider the completion of $\mathcal{D}(\widehat{M})$ with respect to the $L^{p}$-norm constructed out of the metric induced volume form $\mathrm{d} \mu_{\widehat{g}}$. With $\mathcal{E}(\widehat{M})$ we will indicate the space of smooth functions on $\widehat{M}$ endowed with the standard locally convex topology. With $\mathcal{D}^{\prime}(\widehat{M})$ we refer to the space of distributions, whose test functions are the elements of $\mathcal{D}(\widehat{M})$.

Remark 11: The same definitions apply, mutatis mutandis, to the case of $(M, g)$ being a Riemannian manifold with boundary and of bounded geometry, though in this case $\mathcal{D}(M)$ is replaced in the preceding and in the forthcoming discussion by $C_{m e}(M)$, that is the equivalence classes of complex valued measurable functions over $M$. Observe that, in view of Definition [5, we can replace $\mathcal{D}(M)$ also with $\mathcal{D}(\widehat{M}) /\left\{f \in \mathcal{D}(\widehat{M})|f|_{M}=0\right\}$, which is isomorphic to the former.

In order to introduce Sobolev spaces we need suitable local charts. On the one hand, since every Riemannian manifold $(\widehat{M}, \widehat{g})$ of bounded geometry is also complete, we can define the standard geodesic normal coordinates, whose associated atlas is indicated with $\left(U_{\beta}^{\text {geo }}, \kappa_{\beta}^{\text {geo }}\right)_{\beta \in J}, J$ being a suitable index set. If we let $\left\{h_{\beta}^{\text {geo }}\right\}_{\beta \in J}$ be a partition of unity subordinated to this cover we have identified the triple $\mathcal{T}^{\text {geo }} \doteq\left(U_{\beta}^{\text {geo }}, \kappa_{\beta}^{\text {geo }}, h_{\beta}\right)_{\beta \in J}$, which we will refer to as geodesic trivialization of $\widehat{M}$.

On the other hand we say that a cover $\left\{U_{\alpha}\right\}_{\alpha \in I}$ of $\widehat{M}, I$ being an index set, is uniformly locally finite if there exists $n \in \mathbb{N}$ such that each element of the cover is intersected by at most $n$ other sets of the cover. In addition, we consider

1. on each $U_{\alpha}, \alpha \in I$, local coordinates, that is a diffeomorphism $\kappa_{\alpha}: V_{\alpha} \rightarrow U_{\alpha}$, where $V_{\alpha}$ is an open subset of $\mathbb{R}^{m}, m=\operatorname{dim} M$,

2. a partition of unity $\left\{h_{\alpha}\right\}_{\alpha \in I}$ subordinated to the cover $\left\{U_{\alpha}\right\}_{\alpha \in I}$.

The triple $\mathcal{T} \doteq\left(U_{\alpha}, \kappa_{\alpha}, h_{\alpha}\right)_{\alpha \in I}$ is called a uniformly locally finite trivialization of $\widehat{M}$. In the collection of these trivializations, we select a distinguished subclass by the relation with geodesic coordinates:

Definition 12: Let $(\widehat{M}, \widehat{g})$ be a manifold of bounded geometry of dimension $m$. We call $\mathcal{T}$ a uniformly locally finite trivialization of $\widehat{M}$ admissible if the following two conditions are met: 
1. The atlas $\left(U_{\alpha}, k_{\alpha}\right)_{\alpha \in I}$ built out of $\mathcal{T}$ is compatible with a geodesic atlas $\left(U_{\beta}^{\text {geo }}, k_{\beta}^{\text {geo }}\right)_{\beta \in J}$ of $\widehat{M}$, that is, for all $k \in \mathbb{N} \cup\{0\}$, there exists $C_{k}>0$ such that, for all $\alpha \in I$, for all $\beta \in J$ and for all multi-indices $\mathfrak{a} \in(\mathbb{N} \cup\{0\})^{m}$ with $|\mathfrak{a}| \leq k$,

$$
\left|D^{\mathfrak{a}}\left(\kappa_{\alpha}^{-1} \circ \kappa_{\beta}^{\text {geo }}\right)\right| \leq C_{k} \quad \text { and } \quad\left|D^{\mathfrak{a}}\left(\left(\kappa_{\beta}^{\text {geo }}\right)^{-1} \circ \kappa_{\alpha}\right)\right| \leq C_{k},
$$

2. for all $k \in \mathbb{N}$, there exists $c_{k}>0$ such that, for all $\alpha \in I$ and for all multi-indices $\mathfrak{a} \in(\mathbb{N} \cup\{0\})^{m}$ with $|\mathfrak{a}| \leq k$, it holds

$$
\left|D^{\mathfrak{a}}\left(h_{\alpha} \circ \kappa_{\alpha}\right)\right| \leq c_{k}
$$

From now on we shall only consider admissible, uniformly locally finite trivializations.

Definition 13: Let $(\widehat{M}, \widehat{g})$ be a Riemannian manifold of bounded geometry of dimension $m$ and let $\mathcal{T}$ be an admissible, uniformly locally finite trivialization, and let $\mathcal{T}^{\text {geo }}$ be an associated geodesic trivialization. Then, for every $s \in \mathbb{R}$ and for every integer $1<p<\infty$, we call $H_{p}^{s, \mathcal{T}}(\widehat{M})$ the collection of all distributions $u \in \mathcal{D}^{\prime}(\widehat{M})$ such that

$$
\|u\|_{H_{p}^{s, \mathcal{T}}} \doteq\left[\sum_{\alpha \in I}\left\|\left(h_{\alpha} u\right) \circ \kappa_{\alpha}\right\|_{H_{p}^{s}\left(\mathbb{R}^{m}\right)}^{p}\right]^{\frac{1}{p}}<\infty
$$

where $\|\cdot\|_{H_{p}^{s}\left(\mathbb{R}^{m}\right)}$ indicates the standard Sobolev norm on $H_{p}^{s}\left(\mathbb{R}^{m}\right)$. Equivalently we define $H_{p}^{s, \mathcal{T}^{\text {geo }}}(\widehat{M})$ by replacing $\mathcal{T}$ with $\mathcal{T}^{\text {geo }}$.

The following proposition summarizes the results of [GS13, Th. 3.9] and of Section 7.4.5 in [Tri92, see also [Heb96]:

Proposition 14: Let $(\widehat{M}, \widehat{g})$ be a Riemannian manifold of bounded geometry and let $\mathcal{T}, \mathcal{T}^{\text {geo }}$ be respectively a uniformly locally finite and a geodesic trivialization of $\widehat{M}$. Let $k \in \mathbb{N} \cup\{0\}$ and let $W_{p}^{k}(\widehat{M})$ be the completion of the subspace $\left.\mathcal{E}_{p}^{k}(\widehat{M}) \doteq\left\{f \in \mathcal{E}(\widehat{M}) \mid \quad f, \nabla f, \ldots, \nabla^{k} f \in L^{p}(\widehat{M})\right)\right\}$ with respect to the norm

$$
\|f\|_{W_{p}^{k}(\widehat{M})} \doteq\left(\sum_{i=0}^{k}\left\|\nabla^{i} f\right\|_{L^{p}(\widehat{M})}^{p}\right)^{\frac{1}{p}},
$$

$\nabla$ being the covariant derivative built out of the metric $\widehat{g}$. Then it holds that, for all $s \in \mathbb{R}$ and for all integer $p$ such that $1<p<\infty$, neither $H_{p}^{s, \mathcal{T}}(\widehat{M})$ nor $H_{p}^{s, \mathcal{T}^{\text {geo }}}(\widehat{M})$ depend on the choice of the trivialization. Hence $H_{p}^{s}(\widehat{M}) \equiv H_{p}^{s, \mathcal{T}}(\widehat{M})=H_{p}^{s, \mathcal{T}^{\text {geo }}}(\widehat{M})$. In addition, if $s \in \mathbb{N} \cup\{0\}$, it holds that $W_{p}^{s}(\widehat{M})=H_{p}^{s}(\widehat{M})$.

Remark 15: In the preceding discussion we have considered only manifolds without boundary. Nonetheless it is possible to extend or to adapt all definitions also to any Riemannian manifold $(M, g)$ with boundary and of bounded geometry using Definition [13. A detailed discussion has been given especially in AGN16, Sec. 5.1]. In particular we observe that Fermi coordinates as per Definition 8 can be completed to define an admissible trivialization out of which it is possible to define $H_{p}^{s, \mathcal{T}}(M)$ for all $s \in \mathbb{R}$ and for all integer values of $p$ such that $1<p<\infty$. Most notably, it holds, also in this case, that $H_{p}^{s}(M)=W_{p}^{s}(M)$ for all $s \in \mathbb{N} \cup\{0\}$.

Whenever a boundary is present, one can introduce the subspace $H_{0, p}^{s}(M) \subset H_{p}^{s}(M)$ defined as the completion of $\mathcal{D}(M)$ with respect to the $H_{p}^{s}(M)$-norm. Observe that, whenever $M$ is metric complete (e.g. if $M$ is a Riemannian manifold of bounded geometry), $H_{0, p}^{s}(M)=H_{p}^{s}(M)$, while the inclusion is strict in general.

To conclude this section we state the trace theorem, as proven in [GS13, Th. 4.10 \& Cor. 4.12], though specialized to the case of our interest: 
Theorem 16: Let $(M, g)$ be a Riemannian manifold with boundary and of bounded geometry. Let $s \in \mathbb{R}$ and let $H^{s}(M) \doteq H_{2}^{s}(M), H_{0}^{s}(M) \doteq H_{0,2}^{2}(M)$ be the Sobolev spaces as per Definition 13 and per Proposition 14, see also Remark 15. Then, if $s>\frac{1}{2}$, the restriction map from $\mathcal{D}(M)$ to $\mathcal{D}(\partial M)$ extends to a continuous surjective map

$$
\Gamma: H^{s}(M) \rightarrow H^{s-\frac{1}{2}}(\partial M)
$$

If $s \geq 1, \operatorname{ker} \Gamma=H^{s}(M) \cap H_{0}^{1}(M)$.

Observe that, in view of this last theorem, one can read the elements of $H_{0, p}^{s}(M), s \in \mathbb{N}$ as those of $H_{p}^{s}(M)$ whose representatives are functions whose derivatives with order less than $s$ have vanishing trace on $\partial M$.

\section{Boundary triples and their application to second-order elliptic differential operator}

In this section, we consider second-order, elliptic differential operators on a Riemannian manifold with boundary and of bounded geometry, and characterize their self-adjoint extensions. A convenient framework for addressing this question is that of boundary triples, a thoroughly analysed topic of which we recall the main aspects following BL12] and references therein.

\subsection{Boundary triples}

In this section $\mathrm{H}$ indicates a separable Hilbert space over $\mathbb{C}$ while $S: D(S) \subset \mathrm{H} \rightarrow \mathrm{H}$ is a closed, symmetric linear operator.

Definition 17: A boundary triple for the adjoint operator $S^{*}$ is a triple $\left(\mathrm{h}, \gamma_{0}, \gamma_{1}\right)$ consisting of a separable Hilbert space $\mathrm{h}$ over $\mathbb{C}$ and two linear maps $\gamma_{0}, \gamma_{1}: D\left(S^{*}\right) \rightarrow \mathrm{h}$ such that

$$
\left(S^{*} f \mid f^{\prime}\right)_{\mathrm{H}}-\left(f \mid S^{*} f^{\prime}\right)_{\mathrm{H}}=\left(\gamma_{1} f \mid \gamma_{0} f^{\prime}\right)_{\mathrm{h}}-\left(\gamma_{0} f \mid \gamma_{1} f^{\prime}\right)_{\mathrm{h}} \quad \forall f, f^{\prime} \in D\left(S^{*}\right) .
$$

and the map $\gamma: D\left(S^{*}\right) \rightarrow \mathrm{h} \times \mathrm{h}$, defined by $\gamma(f)=\left(\gamma_{0}(f), \gamma_{1}(f)\right)$ for all $f \in D\left(S^{*}\right)$, is surjective.

Example 18: A canonical example of boundary triple, sometimes termed ordinary boundary triple, can be constructed if $S$ has equal and finite deficiency indices $d_{ \pm}(S)=\operatorname{dim}\left(\mathcal{N}_{ \pm}\left(S^{*}\right)\right)<\infty, \mathcal{N}_{ \pm}\left(S^{*}\right) \doteq$ $\operatorname{ker}\left(S^{*} \pm i \mathbb{I}\right)$, hence admitting self-adjoint extensions. In this case, letting $V: \mathcal{N}_{-}\left(S^{*}\right) \rightarrow \mathcal{N}_{+}\left(S^{*}\right)$ be any, but fixed unitary operator, one can set $\mathrm{h}=\mathcal{N}_{+}\left(S^{*}\right)$. Since $D\left(S^{*}\right)=D(S) \oplus_{S} \mathcal{N}_{+}\left(S^{*}\right) \oplus_{S} \mathcal{N}_{-}\left(S^{*}\right) 2$, one can introduce the projection maps $\pi_{ \pm}: D\left(S^{*}\right) \rightarrow \mathcal{N}_{ \pm}\left(S^{*}\right)$, defining $\gamma_{0} \doteq \pi_{+}-V \circ \pi_{-}$and $\gamma_{1} \doteq i\left(\pi_{+}+V \circ \pi_{-}\right)$. Observe that, since $V$ is a bijection, the map $\gamma$ as in Definition 17 is automatically surjective. Finally, equation (4) follows by the identity

$$
\left(S^{*} f \mid f^{\prime}\right)-\left(f \mid S^{*} f^{\prime}\right)=2 i\left[\left(\pi_{-} f \mid \pi_{-} f^{\prime}\right)_{\mathrm{H}}-\left(\pi_{+} f \mid \pi_{+} f^{\prime}\right)_{\mathrm{H}}\right],
$$

which holds true for all $f, f^{\prime} \in D\left(S^{*}\right)$.

Boundary triples allow us to characterize the self-adjoint extensions of $S$ in terms of "boundary conditions" on h. As a starting point one can observe, that, under the hypotheses of Definition [17, one can single out two distinguished self-adjoint extensions of $S$, namely

$$
\left.S_{0} \doteq S^{*}\right|_{\operatorname{ker} \gamma_{0}},\left.\quad S_{1} \doteq S^{*}\right|_{\operatorname{ker} \gamma_{1}}
$$

\footnotetext{
${ }^{2}$ The orthogonal decomposition $\oplus_{S}$ refers to the scalar product on $D\left(S^{*}\right)$ defined by $\left(f \mid f^{\prime}\right)_{S}:=\left(f \mid f^{\prime}\right)+\left(S^{*} f \mid S^{*} f^{\prime}\right)$.
} 
which are transversal since $D(S)=D\left(S_{0}\right) \cap D\left(S_{1}\right)$ and $D\left(S^{*}\right)=D\left(S_{0}\right)+D\left(S_{1}\right)$, + standing for the algebraic sum. The following proposition shows how to construct all other sought extensions, $c f$. [Mal92] for a proof:

Proposition 19: Let $S$ be a closed, symmetric operator on $\mathrm{H}$. Then an associated boundary triple (h, $\gamma_{0}, \gamma_{1}$ ) exists if and only if $S^{*}$ has equal deficiency indices. In addition, if $\Theta: D(\Theta) \subseteq \mathrm{h} \rightarrow \mathrm{h}$ is a closed and densely defined linear operator, then $\left.S_{\Theta} \doteq S^{*}\right|_{\operatorname{ker}\left(\gamma_{1}-\Theta \gamma_{0}\right)}$ is a closed extension of $S$ with domain

$$
D\left(S_{\Theta}\right) \doteq\left\{f \in D\left(S^{*}\right) \mid \gamma_{0}(f) \in D(\Theta), \gamma_{1}(f)=\Theta \gamma_{0}(f)\right\}
$$

The map $\Theta \mapsto S_{\Theta}$ is one-to-one and $S_{\Theta}^{*}=S_{\Theta^{*}}$, that is, it restricts to a one-to-one map from self-adjoint operators $\Theta$ to self-adjoint extensions of $S$.

Observe that, in this formulation, $S_{1}$ can be recovered by setting $\Theta=0$, while $S_{0}$ represents a kind of degenerate scenario, which has to be included by hand. The reason is due to the formulation of the proposition which we have chosen so to emphasize the connection with the heuristic notion of boundary conditions.

Example 20: With reference to Example 18, self-adjoint extensions of $S$ are in one-to-one correspondence with surjective isometries $U: \mathcal{N}_{-}\left(S^{*}\right) \rightarrow \mathcal{N}_{+}\left(S^{*}\right)$, cf. [Mor18, Th. 5.37], the domain of the extension being $D\left(S_{U}\right)=D(S) \oplus_{S}(\mathbb{I}-U) \mathcal{N}_{-}\left(S^{*}\right)$. At the same time Proposition 19 guarantees that all self-adjoint extensions of $S$ are completely determined by the self-adjoint operators $\Theta: \mathrm{h} \rightarrow \mathrm{h}$. Since the domain of the extension $S_{\Theta}$ is the collection of elements $f \in D\left(S^{*}\right)$ such that $\gamma_{1}(f)=\Theta \gamma_{0}(f)$, this is equivalent to requiring $(i \mathbb{I}-\Theta) \pi_{+}(f)=-(i \mathbb{I}+\Theta) V \circ \pi_{-}(f)$. In other words $\pi_{+}(f)=\mathcal{C}(-\Theta) V \circ \pi_{-}(f)$, where $\mathcal{C}(-\Theta)$ stands for the Cayley transform of $\Theta$, hence a unitary operator on $\mathrm{h}, c f$. [Mor18, Th. 5.34]. By identifying $U=\mathrm{C}(-\Theta) V$ we have shown how to relate the two viewpoints.

To conclude this short digression, we emphasize how boundary triples also allow to obtain the spectral properties of the self-adjoint extensions of an Hermitian operator $S$ as above. To this end, first we need an auxiliary lemma, BL12

Lemma 21: Let $S: D(S) \subseteq \mathrm{H} \rightarrow \mathrm{H}$ be a closed, symmetric operator and let $\left(\mathrm{h}, \gamma_{0}, \gamma_{1}\right)$ be an associated boundary triple. Let $S^{\prime}$ be any self-adjoint extension of $S, S \subset S^{\prime} \subset S^{*}$, and let $\lambda \in \rho\left(S^{\prime}\right), \rho$ being the resolvent set. Then 3

$$
D\left(S^{*}\right)=D\left(S^{\prime}\right) \dot{+} \mathcal{N}_{\lambda}\left(S^{*}\right), \quad \mathcal{N}_{\lambda}\left(S^{*}\right) \doteq \operatorname{ker}\left(S^{*}-\lambda \mathbb{I}\right) .
$$

Proof. Let $f \in D\left(S^{*}\right)$ and let $\tilde{f}=\left(S^{\prime}-\lambda \mathbb{I}\right)^{-1}\left(\left(S^{*}-\lambda \mathbb{I}\right) f\right) \in D\left(S^{\prime}\right)$, where $\lambda \in \rho\left(S^{\prime}\right)$. For any $f^{\prime} \in D(S)$ it holds that

$$
\begin{aligned}
\left(\left(S^{\prime}-\bar{\lambda} \mathbb{I}\right) f^{\prime}, f-\tilde{f}\right)_{\mathrm{H}} & =\left(\left(S^{\prime}-\bar{\lambda} \mathbb{I}\right) f^{\prime}, f\right)_{\mathrm{H}}-\left(\left(S^{\prime}-\bar{\lambda} \mathbb{I}\right) f^{\prime},\left(S^{\prime}-\lambda \mathbb{I}\right)^{-1}\left(\left(S^{*}-\lambda \mathbb{I}\right) f\right)\right)_{\mathrm{H}} \\
& =\left(\left(S^{\prime}-\bar{\lambda} \mathbb{I}\right) f^{\prime}, f\right)_{\mathrm{H}}-\left(f^{\prime},\left(S^{*}-\lambda \mathbb{I}\right) f\right)_{\mathrm{H}}=0
\end{aligned}
$$

where the last equality descends by using that $S^{* *}=S$, being $S$ closed. Hence $f-\tilde{f} \in \operatorname{Ran}(S-\bar{\lambda} \mathbb{I})^{\perp}=$ $\operatorname{ker}\left(S^{*}-\lambda \mathbb{I}\right)=\mathcal{N}_{\lambda}\left(S^{*}\right)$. We have found the sought decomposition. To prove that it is unique, it suffices to observe that $D\left(S^{\prime}\right) \cap \mathcal{N}_{\lambda}\left(S^{*}\right)=\{0\}$ since $\lambda \in \rho\left(S^{\prime}\right)$ per assumption.

We define the following auxiliary functions:

\footnotetext{
${ }^{3}$ In this paper $V+W$ denotes the direct sum between the subspaces $V, W \subseteq \mathbf{H}(V+W=\mathbf{H}$ and $V \cap W=\{0\})$ while $V \oplus W$ denotes the orthogonal direct sum $(V+W=\mathrm{H}$ and $V \perp W)$.
} 
Definition 22: Let $S: D(S) \subseteq \mathrm{H} \rightarrow \mathrm{H}$ be a closed, symmetric operator and let $\left(\mathrm{h}, \gamma_{0}, \gamma_{1}\right)$ be an associated boundary triple. Moreover, consider the self-adjoint extension $S_{0}$ of $S$ defined by $\left.S_{0} \doteq S^{*}\right|_{\text {ker } \gamma_{0}}$. We call $\gamma$-field and Weyl function respectively the maps $\Gamma: \rho\left(S_{0}\right) \rightarrow D\left(S^{*}\right)$ and $M: \rho\left(S_{0}\right) \rightarrow \mathrm{h}$ such that

$$
\Gamma(\lambda) \doteq\left[\left.\gamma_{0}\right|_{\mathcal{N}_{\lambda}\left(S^{*}\right)}\right]^{-1}, \quad M(\lambda) \doteq \gamma_{1} \circ \Gamma(\lambda),
$$

where $\rho\left(S_{0}\right)$ is the resolvent of $S_{0}$.

We have all the ingredients to state the following key theorem which specializes to the case at hand a statement proven in DM91:

Theorem 23: Let $S: D(S) \subseteq \mathrm{H} \rightarrow \mathrm{H}$ be a closed, symmetric operator and let $\left(\mathrm{h}, \gamma_{0}, \gamma_{1}\right)$ be an associated boundary triple. Let $S_{\Theta}$ be a self-adjoint extension of $S$ determined by means of a self-adjoint operator $\Theta: D(\Theta) \subseteq \mathrm{h} \rightarrow \mathrm{h}$. Let $\rho, \sigma_{p}$ and $\sigma_{c}$ indicate respectively resolvent, point and continuous spectrum of an operator. Then for every $\lambda \in \rho\left(S_{0}\right), S_{0}=\left.D\left(S^{*}\right)\right|_{\operatorname{ker} \gamma_{0}}$, it holds

1. $\lambda \in \rho\left(S_{\Theta}\right)$ if and only if $0 \in \rho(\Theta-M(\lambda))$, where $M$ is the Weyl function,

2. $\lambda \in \sigma_{i}\left(S_{\Theta}\right), i=p, c$ if and only if $0 \in \sigma_{i}(\Theta-M(\lambda))$.

In other words, this theorem guarantees that the computation of the spectrum of $S_{\Theta}$, a self-adjoint extension of $S$, boils down to evaluating the spectra of $S_{0}$ and of $\Theta-M(\lambda)$.

\subsection{Application to second-order elliptic differential operators}

In this section we apply the theory of boundary triples to the study of second-order, elliptic differential operators. We start our analysis from the distinguished, albeit special case of the Laplace-Beltrami operator, subsequently generalizing our construction. Hence, let $(M, g)$ be a Riemannian manifold with boundary and of bounded geometry as per Definition [5. On top of $M$ we consider the Laplace-Beltrami operator $\Delta_{g}$, which reads in a local chart $\Delta_{g}=-\nabla_{i} g^{i j} \nabla_{j}, \nabla$ being the Levi-Civita connection built out of $g$. We wish to identify a boundary triple for $\Delta_{g}$, which is regarded as a densely defined operator $\Delta_{g}: H_{0}^{2}(M) \rightarrow L^{2}\left(M ; d \mu_{g}\right), H_{0}^{2}(M)$ being the closure of $\mathcal{D}(M)$ with respect to the $H^{2}(M)$-norm as defined in Proposition 14, see also Remark 15. Standard arguments yield that $\Delta_{g}$ is a closed symmetric operator on $L^{2}(M) \equiv L^{2}\left(M ; d \mu_{g}\right)$ whose adjoint $\Delta_{g}^{*}$ is defined on the so-called maximal domain

$$
D_{\max }\left(\Delta_{g}^{*}\right) \doteq\left\{f \in L^{2}(M) \mid \Delta_{g}(f) \in L^{2}(M)\right\}, \quad \Delta_{g}^{*} f \doteq \Delta_{g} f .
$$

Since $(M, g)$ is of bounded geometry, then $\Delta_{g}$ is uniformly elliptic and the maximal domain coincides with $H^{2}(M)$. Hence we shall identify $D_{\max }\left(\Delta_{g}^{*}\right)=H^{2}(M)$.

To construct a boundary triple associated with $\Delta_{g}^{*}$, let $n$ be the outward-pointing unit normal of $\partial M$ and let

$$
\Gamma_{0}: H^{2}(M) \ni f \mapsto \Gamma f \in H^{3 / 2}(\partial M), \quad \Gamma_{1}: H^{2}(M) \ni f \mapsto-\Gamma \nabla_{n} f \in H^{1 / 2}(\partial M),
$$

where $\Gamma$ is the trace map defined in Theorem[16. For $f_{1}, f_{2} \in H^{2}(M)=D_{\max }\left(\Delta_{g}^{*}\right)$, the following Green's identity holds true:

$$
\left(\Delta_{g}^{*} f_{1} \mid f_{2}\right)_{L^{2}(M)}-\left(f_{1} \mid \Delta_{g}^{*} f_{2}\right)_{L^{2}(M)}=\left(\Gamma_{1} f_{1} \mid \Gamma_{0} f_{2}\right)_{L^{2}(\partial M)}-\left(\Gamma_{0} f_{1} \mid \Gamma_{1} f_{2}\right)_{L^{2}(\partial M)} .
$$

Moreover, since the inner product $(\mid)_{L^{2}(\partial M)}$ on $L^{2}(\partial M) \equiv L^{2}\left(\partial M ; \iota_{M}^{*} d \mu_{g}\right), \iota_{M}: \partial M \hookrightarrow M$, extends continuously to a pairing on $H^{-1 / 2}(\partial M) \times H^{1 / 2}(\partial M)$ as well as on $H^{-3 / 2}(\partial M) \times H^{3 / 2}(\partial M)$, there exist isomorphisms

$$
j_{ \pm}: H^{ \pm 1 / 2}(\partial M) \rightarrow L^{2}(\partial M), \quad \iota_{ \pm}: H^{ \pm 3 / 2}(\partial M) \rightarrow L^{2}(\partial M)
$$


such that, for all $(\psi, \phi) \in H^{1 / 2}(\partial M) \times H^{-1 / 2}(\partial M)$ and for all $(\widetilde{\psi}, \widetilde{\phi}) \in H^{3 / 2}(\partial M) \times H^{-3 / 2}(\partial M)$,

$$
(\psi, \phi)_{(1 / 2,-1 / 2)}=\left(j_{+} \psi \mid j_{-} \phi\right)_{L^{2}(\partial M)}, \quad(\widetilde{\psi}, \widetilde{\phi})_{(3 / 2,-3 / 2)}=\left(\iota_{+} \widetilde{\psi} \mid \iota_{-} \widetilde{\phi}\right)_{L^{2}(\partial M)},
$$

where $(,)_{(1 / 2,-1 / 2)}$ and $(,)_{(3 / 2,-3 / 2)}$ stand for the duality pairings between the associated Sobolev spaces.

Gathering all the above ingredients, the following result holds, being a generalization to the case of Riemannian manifolds with boundary and of bounded geometry of a classical result, e.g. Gru68:

Proposition 24: Let $\Delta_{g}^{*}$ be the adjoint of the Laplace-Beltrami operator on a Riemannian manifold $(M, g)$ with boundary and of bounded geometry. Let

$$
\begin{aligned}
& \gamma_{0}: H^{2}(M) \ni f \mapsto \iota_{+} \Gamma_{0} f \in L^{2}(\partial M), \\
& \gamma_{1}: H^{2}(M) \ni f \mapsto j_{+} \Gamma_{1} f \in L^{2}(\partial M),
\end{aligned}
$$

Then $\left(L^{2}(\partial M), \gamma_{0}, \gamma_{1}\right)$ is a boundary triple for $\Delta_{g}^{*}$. Moreover the self adjoint extension $\left.\Delta_{g, 0} \doteq \Delta_{g}^{*}\right|_{\operatorname{ker} \gamma_{0}}$ coincides with the Dirichlet operator $\left.\Delta_{g}^{*}\right|_{H^{2}(M) \cap H_{0}^{1}(M)}$.

Proof. It suffices to observe that, in view of our assumption on the geometry of $(M, g)$, the trace map $\Gamma$ is both continuous and surjective, c.f GS13. Hence also the map $\gamma: H^{2}(M) \rightarrow L^{2}(\partial M) \times L^{2}(\partial M)$ is surjective by combining the properties of $\Gamma$ together with the fact that $\iota_{+}$and $j_{+}$are isomorphisms and $\nabla_{n}: H^{s}(M) \rightarrow H^{s-1}(M)$. Together with this data one can repeat the same proof as in Gru68 in combination with a partition of unity argument. The last statement of this proposition descends from Theorem 16 and, in particular from the kernel of $\Gamma$ acting on $H^{s}(M)$ being $H_{0}^{1}(M) \cap H^{s}(M)$ for all $s \geq 1$.

Remark 25: Notice that Proposition 24 ensures that the self-adjoint extensions of $\Delta_{g}$ are parametrized by all densely defined self-adjoint operators $\Theta$ on $L^{2}(\partial M)$. Therefore, the class of boundary conditions $\operatorname{ker}\left(\gamma_{1}-\Theta \gamma_{0}\right)$ is more general then the one considered usually in the literature [Hör90].

Remark 26: We observe that, while the results of Proposition 24 can be straightforwardly extended if one adds to $\Delta_{g}$ terms of order 0 which are both smooth and bounded, dealing with a generic elliptic differential operator $A$ of order 2 on $(M, g)$ requires a more careful investigation.

More specifically consider an operator $A$ which reads locally $A=-\nabla_{i} a^{i j} \nabla_{j}$, where both $a^{i j}$ and $\nabla_{i} a^{i j} \in C^{\infty}(M) \cap L^{\infty}(M)$. One has individuated a closed, symmetric operator $A: H_{0}^{2}(M) \rightarrow L^{2}(M)$ whose adjoint $A^{*}$ has a maximal domain

$$
D\left(A^{*}\right) \doteq\left\{f \in L^{2}(M) \mid A f \in L^{2}(M)\right\}, \quad A^{*} f \doteq A f .
$$

Since $(M, g)$ is of bounded geometry, $H^{2}(M) \subseteq D\left(A^{*}\right)$, though the inclusion is in general strict unless $A$ is uniformly elliptic. Since $H^{2}(M)$ is dense in $D\left(A^{*}\right)$, see ILP13 and references therein, the maps

$$
\left(\Gamma_{0}, \Gamma_{1}\right): H^{2}(M) \ni f \mapsto\left(\Gamma f,-\Gamma \nabla_{a, n} f\right) \in H^{1 / 2}(\partial M) \times H^{3 / 2}(\partial M),
$$

where $\nabla_{a, n} f \doteq n_{i} a^{i j} \nabla_{j} f$ while $\Gamma$ is defined as in Theorem (16), can be extended to linear continuous maps

$$
\left(\widetilde{\Gamma}_{0}, \widetilde{\Gamma}_{1}\right): D\left(A^{*}\right) \rightarrow H^{-1 / 2}(\partial M) \times H^{-3 / 2}(\partial M) .
$$

In addition, if $f_{1} \in D\left(A^{*}\right)$ and $f_{2} \in H^{2}(M)$, it holds

$$
\begin{aligned}
\left(A^{*} f_{1} \mid f_{2}\right)_{L^{2}(M)}-\left(f_{1} \mid A^{*} f_{2}\right)_{L^{2}(M)} & =\left(\widetilde{\Gamma}_{1} f_{1} \mid \Gamma_{0} f_{2}\right)_{L^{2}(\partial M)}-\left(\widetilde{\Gamma}_{0} f_{1} \mid \Gamma_{1} f_{2}\right)_{L^{2}(\partial M)} \\
& =\left(\iota_{-} \widetilde{\Gamma}_{1} f_{1} \mid \iota_{+} \Gamma_{0} f_{2}\right)_{-\frac{3}{2}, \frac{3}{2}}-\left(j_{-} \widetilde{\Gamma}_{0} f_{1} \mid j_{+} \Gamma_{1} f_{2}\right)_{-\frac{1}{2}, \frac{1}{2}},
\end{aligned}
$$


where in the last equality we used the definition of $\iota_{ \pm}, j_{ \pm}$while $(,)_{-\frac{3}{2}, \frac{3}{2}},(,)_{-\frac{1}{2}, \frac{1}{2}}$ denote the pairing between dual spaces. In order for the above data to define a boundary triple, let $A_{0}$ be the Dirichlet self-adjoint extension of $A$, defined as $\left.A_{0} \doteq A^{*}\right|_{H^{2}(M) \cap H_{0}^{1}(M)}$. By applying Lemma 21 for $S^{*}=A^{*}$ and for $S^{\prime}=A_{0}$ it follows that, for an arbitrary but fixed $\lambda \in \rho\left(A_{0}\right)$, the maximal domain $D\left(A^{*}\right)$ decomposes as

$$
D\left(A^{*}\right)=D\left(A_{0}\right) \dot{+} \operatorname{ker}\left(A^{*}-\lambda\right) .
$$

One can consider the maps

$$
\left(\gamma_{0}, \gamma_{1}\right): D\left(A^{*}\right) \ni f \mapsto\left(j_{-} \widetilde{\Gamma}_{0} f_{\lambda}, \iota_{+} \widetilde{\Gamma}_{1} f_{0}\right)=\left(j_{-} \widetilde{\Gamma}_{0} f_{\lambda}, \iota_{+} \Gamma_{1} f_{0}\right) \in L^{2}(\partial M) \times L^{2}(\partial M),
$$

where $f_{0} \in D\left(A_{0}\right)$ is the "Dirichlet part" of $f=f_{0}+f_{\lambda}$ according to decomposition (10), while $f_{\lambda} \in$ $\operatorname{ker}\left(S^{*}-\lambda\right)$. It can be shown that $\left(L^{2}(\partial M), \gamma_{0}, \gamma_{1}\right)$ is a boundary triple for $A^{*}$ HSF12, Gru71, moreover, the self adjoint extension $\left.S_{0} \doteq S^{*}\right|_{H^{2}(M) \cap H_{0}^{1}(M)}$ coincides with the self-adjoint extension $\left.S^{*}\right|_{\text {ker } \gamma_{0}}$, hence justifying the notation. We remark that the ambiguity in the choice of $\lambda \in \rho\left(S_{0}\right)$ reflects possible degeneracies of $A$.

\section{$3 \quad$ Fundamental solutions on spacetimes with timelike boundary}

In this section, following Section 1.1 and Proposition 9 in particular, we consider $(N, \widetilde{h})$ to be a static Lorentzian spacetime with timelike boundary. Henceforth, for any $\Omega \subseteq N$, with $J^{ \pm}(\Omega)$ we indicate the causal future $(+)$ and the causal past $(-)$ of $\Omega$, cf. [BEE96, Ch. 1]. On top of it we consider the D'Alembert wave operator which, in view of (1), reads locally

$$
\square_{\widetilde{h}}=-\widetilde{h}^{i j} \nabla_{i} \nabla_{j}=\beta^{-1} \partial_{t}^{2}-\frac{1}{2} g^{i j} \partial_{i}(\ln \beta) \partial_{j}+\Delta_{g},
$$

where $\Delta_{g}$ is the Laplace-Beltrami operator associated with $(M, g)$. The solutions of the wave equation built out of (12) are best characterized in terms of the fundamental solutions associated with $\square_{\tilde{h}}$.

Definition 27: A continuous linear map $\widetilde{\mathrm{G}}^{ \pm}: \mathcal{D}(\stackrel{\circ}{N}) \rightarrow \mathcal{D}^{\prime}(\stackrel{\circ}{N})$ is called a retarded $(+)$ or an advanced $(-)$ Green's operator for $\square_{\widetilde{h}}$ on a static Lorentzian spacetime $(N, h)$ with timelike boundary if, for every $f \in \mathcal{D}(\stackrel{\circ}{N}), \stackrel{\circ}{N} \equiv N \backslash \partial N$,

$$
\square_{\widetilde{h}} \circ \widetilde{\mathrm{G}}^{ \pm} f=\widetilde{\mathrm{G}}^{ \pm} \circ \square_{\widetilde{h}} f=f, \quad \operatorname{supp}\left(\widetilde{\mathrm{G}}^{ \pm} f\right) \subseteq J^{\mp}(\operatorname{supp}(f)) .
$$

The causal (or advanced-minus-retarded) propagator $\widetilde{\mathrm{G}}$ associated to the pair $\left(\widetilde{\mathrm{G}}^{+}, \widetilde{\mathrm{G}}^{-}\right)$is the linear operator

$$
\widetilde{\mathrm{G}} \doteq \widetilde{\mathrm{G}}^{-}-\widetilde{\mathrm{G}}^{+}: \mathcal{D}(\stackrel{\circ}{N}) \rightarrow \mathcal{D}^{\prime}(\stackrel{\circ}{N})
$$

Remark 28: In view of Proposition 9, under mild additional hypotheses, $(N, \widetilde{h})$ can be realized as a submanifold of a standard static, globally hyperbolic spacetime $(\widehat{N}, \widehat{h})$. Thereon one can consider the D'Alembert wave operator $\square_{\widehat{h}}$ and standard results in the theory of normally hyperbolic operators guarantee the existence of unique advanced/retarded fundamental solutions $\widehat{\mathrm{G}}^{ \pm}$with the same defining properties as in (14) and, in addition, $\widehat{\mathrm{G}}^{ \pm}[\mathcal{D}(\widehat{N})] \subset C^{\infty}(\widehat{N})$. As a consequence, whenever $\Omega \subset \stackrel{\circ}{N}$ endowed with $\left.\widehat{h}\right|_{\Omega}$ identifies a globally hyperbolic submanifold of $N$ and thus also of $\widehat{N}$, it turns out that, calling $\widetilde{\mathrm{G}}_{\Omega}^{ \pm}$ 
the fundamental solutions for the D'Alembert wave operator on $\left(\Omega,\left.\widehat{h}\right|_{\Omega}\right)$, then $\left.\widehat{\mathrm{G}}^{ \pm}\right|_{\Omega}=\widetilde{\mathrm{G}}_{\Omega}^{ \pm}$and, whenever $\widetilde{\mathrm{G}}^{ \pm}$exist, $\left.\widetilde{\mathrm{G}}^{ \pm}\right|_{\Omega}=\widetilde{\mathrm{G}}_{\Omega}^{ \pm}$. The same line of reasoning cannot be used to compare $\widehat{\mathrm{G}}^{ \pm}$with $\widetilde{\mathrm{G}}^{ \pm}$since $N$ is not a globally hyperbolic submanifold of $\widehat{N}$. We will be showing that the existence and uniqueness of $\widetilde{\mathrm{G}}^{ \pm}$is instead subordinated to the choice of 'boundary conditions' at $\partial N$.

Conformal reduction. In order to address the problem of the existence of $\widetilde{\mathrm{G}}^{ \pm}$, it is convenient to consider on $(N, \widetilde{h})$ the conformally rescaled metric

$$
h \doteq \beta^{-1} \widetilde{h}=-\mathrm{d} t^{2}+\beta^{-1} g,
$$

whose wave operator reads $\square_{h}=\partial_{t}^{2}+\Delta_{\beta^{-1} g}$. The connection between these wave operators is well known, in particular

$$
\square_{\widetilde{h}} \circ \beta^{\frac{1-m}{4}}=\beta^{-\frac{3+m}{4}}\left[\square_{h}+\frac{1-m}{2} \beta^{-\frac{1}{2}} \square_{h}\left(\beta^{\frac{1}{2}}\right)-\frac{(1-m)(m-3)}{4} \beta h^{i j} \nabla_{i} \beta \nabla_{j} \beta\right] \doteq \beta^{-\frac{3+m}{4}}\left[\partial_{t}^{2}+A\right],
$$

where $\operatorname{dim} N=m+1$ while $A$ is an elliptic operator as per remark 26, In the following we will give a construction for the advanced and retarded Green's operators $\mathrm{G}^{ \pm}$for $\partial_{t}^{2}+A$ rather that for $\square_{\tilde{h}}$. Its counterparts, namely $\widetilde{\mathrm{G}}^{ \pm}$, are related to the former ones via the identity

$$
\widetilde{\mathrm{G}}^{ \pm}=\beta^{\frac{1-m}{4}} \circ \mathrm{G}^{ \pm} \circ \beta^{\frac{3+m}{4}} .
$$

In the following our results will depend on the regularity properties of $\beta$ and in particular we shall select it in such a way that the spacetime $(N, h)$ meets the conditions of Proposition 9, where $h=-\mathrm{d} t^{2}+\beta^{-1} g$. This entails in particular that the operator

$$
A \doteq \Delta_{\beta^{-1} g}+\frac{1-m}{2} \beta^{-\frac{1}{2}} \Delta_{\beta^{-1} g}\left(\beta^{\frac{1}{2}}\right)-\frac{(1-m)(m-3)}{4} g^{i j} \nabla_{i}(\beta) \nabla_{j}(\beta),
$$

is uniformly elliptic.

In order to tackle the problem of the existence of $\mathrm{G}^{ \pm}$as per Definition 27 one can use Schwartz kernel theorem, rewriting the underlying problem in terms of two distributions $\mathcal{G}^{ \pm} \in \mathcal{D}^{\prime}(\stackrel{\circ}{N} \times \stackrel{\circ}{N})$ such that, besides the condition on the support stated in (13),

$$
\left[\left(\partial_{t}^{2}+A\right) \otimes \mathbb{I}\right] \mathcal{G}^{ \pm}=\left[\mathbb{I} \otimes\left(\partial_{t}^{2}+A\right)\right] \mathcal{G}^{ \pm}=\delta_{\operatorname{diag}(\stackrel{\circ}{ })} .
$$

Here $\delta_{\text {diag }(N)}$ being the Dirac-delta distribution on the diagonal $\operatorname{diag}(\stackrel{\circ}{N}) \stackrel{\iota}{\hookrightarrow} \stackrel{\circ}{N} \times \stackrel{\circ}{N}$ of $\stackrel{\circ}{N}^{\circ} \stackrel{\circ}{N}$ such that, for every $f \in \mathcal{D}(\stackrel{\circ}{N} \times \stackrel{\circ}{N}), \delta_{\operatorname{diag}(\stackrel{\circ}{N})}(f)=\int_{N} \iota^{*} f \mathrm{~d} \mu_{h}$, where $\mathrm{d} \mu_{h}$ is the metric induced volume form on $N$. Since $(N, h)$ is per assumption a standard static spacetime, realizing it as the warped product $M \times{ }_{\beta} \mathbb{R}$, we can make the ansatz $\mathcal{G}^{-}=\theta\left(t-t^{\prime}\right) \mathcal{G}$ and $\mathcal{G}^{+}=-\theta\left(t^{\prime}-t\right) \mathcal{G}$, where $\theta$ stands for the Heaviside distribution on $\mathbb{R} \times \mathbb{R}$ which is implicitly extended to $N \times \stackrel{\circ}{N}$. From (19) it turns out that $\mathcal{G} \in \mathcal{D}^{\prime}(\stackrel{\circ}{N} \times \stackrel{\circ}{N})$ is the distribution associated to $\mathrm{G}$ via Schwartz kernel theorem and it satisfies

$$
\left\{\begin{array}{l}
{\left[\left(\partial_{t}^{2}+A\right) \otimes \mathbb{I}\right] \mathcal{G}=\left[\mathbb{I} \otimes\left(\partial_{t}^{2}+A\right)\right] \mathcal{G}=0} \\
\left.\mathcal{G}\right|_{t=t^{\prime}}=0,\left.\quad \partial_{t} \mathcal{G}\right|_{t=t^{\prime}}=-\left.\partial_{t^{\prime}} \mathcal{G}\right|_{t=t^{\prime}}=\delta_{\operatorname{diag}(M)}
\end{array},\right.
$$

where $\left.\right|_{t=t^{\prime}}$ indicates the pull-back of a distribution on $\stackrel{\circ}{N} \times \stackrel{\circ}{N}$ to $(\{t\} \times \stackrel{\circ}{M}) \times(\{t\} \times \stackrel{\circ}{M})$ while $\delta_{\operatorname{diag}(\stackrel{\circ}{M})}$ stands for the Dirac delta distribution on the diagonal of $\stackrel{M}{\circ} \times \stackrel{\circ}{M}$. Observe that the product between the Heaviside distribution and $\mathcal{G}$ which defines $\mathcal{G}^{ \pm}$is well-posed as a consequence of [Hör90, Th. 8.2.10]. 
Remark 29: From the previous discussion, it emerges that the existence of the Green's operators $\mathrm{G}^{ \pm}$can be reduced to finding first a solution for (20) and proving, subsequently, that the support properties in (13) are verified. In order to motivate the strategy that we shall follow to address these two questions for static Lorentzian spacetimes with timelike boundary, let us consider a special instance of the scenario of Remark 28. $(N, h)$ is realized as a submanifold of a globally hyperbolic, standard static spacetime $(\widehat{N}, \widehat{h})$ where the warping factor $\widehat{\beta}$ of Proposition 9 is assumed to be equal to 1 . Hence $\widehat{N}$ is isometric to $\widehat{M} \times \mathbb{R}$ where $(\widehat{M}, \widehat{g})$ is a complete Riemannian manifold. The d'Alembert wave operator reads $\square_{\widehat{h}}=\partial_{t}^{2}+\Delta_{\widehat{g}}$. In this framework the Laplace-Beltrami operator $\Delta_{\widehat{g}}$ is known to be an essentially self-adjoint operator on $L^{2}(\widehat{M}) \equiv L^{2}\left(\widehat{M} ; \mathrm{d} \mu_{\widehat{g}}\right), \mathrm{d} \mu_{\widehat{g}}$ being the metric induced volume form. Indicating with a slight abuse of notation still with $\Delta_{\widehat{g}}$ the unique self-adjoint extension, for every $f \in \mathcal{D}(\widehat{M} \times \mathbb{R})$ it holds

$$
\widehat{\mathcal{G}}\left(f_{1}, f_{2}\right)=\int_{\mathbb{R}^{2}}\left(f_{1}(t) \mid \Delta_{\widehat{g}}^{-\frac{1}{2}} \sin \left[\Delta_{\widehat{g}}^{\frac{1}{2}}\left(t-t^{\prime}\right)\right] f_{2}\left(t^{\prime}\right)\right) \mathrm{d} t \mathrm{~d} t^{\prime},
$$

where $f(t) \in H^{2}(M)$ denotes the evaluation of $f$, regarded as an element of $C_{\mathrm{c}}^{\infty}\left(\mathbb{R}, H^{2}(\widehat{M})\right)$. Here $\Delta_{\widehat{g}}^{-\frac{1}{2}} \sin \left[\Delta_{\widehat{g}}^{\frac{1}{2}}\left(t-t^{\prime}\right)\right]$ in a densely defined self-adjoint operator on $L^{2}(M)$ which is defined exploiting the functional calculus for $\widehat{\Delta}_{g}$ [ReSi81, Chap. 7].

From the example outlined in this last remark, it turns out that a solution of (20) can be identified by exploiting the boundary triples introduced in the previous section. Indeed, by fixing a boundary condition $\Theta$ we are reduced to dealing with the operator $\partial_{t}^{2}+A_{\Theta}$ for which we can exploit the spectral calculus associated with $A_{\Theta}$ as in the previous remark 29. This procedure will define a causal propagator for $\partial_{t}^{2}+A$ which can also be regarded as the unique causal propagator for $\partial_{t}^{2}+A_{\Theta}$. The following theorem translates this paradigm:

Theorem 30: Let $(N, h)$ be a static Lorentzian spacetime with timelike boundary as per Proposition 9 . Let $\left(\gamma_{0}, \gamma_{1}, L^{2}(\partial M)\right)$ be the boundary triple as in (11) associated with the elliptic operator $A^{*}$ defined in (18) and let $\Theta$ be a densely defined self-adjoint operator on $L^{2}(\partial M)$. Let $A_{\Theta}$ be the self-adjoint extension of $A$ defined as per Proposition 19 by $\left.A_{\Theta} \doteq A^{*}\right|_{D\left(A_{\Theta}\right)}$, where $D\left(A_{\Theta}\right) \doteq \operatorname{ker}\left(\gamma_{1}-\Theta \gamma_{0}\right)$. Furthermore, let assume that the spectrum of $A_{\Theta}$ is bounded from below.

Then the advanced and retarded Green's operators $\mathrm{G}_{\Theta}^{ \pm}$associated to the wave operator $\partial_{t}^{2}+A_{\Theta}$ exist and they are unique. They are completely determined in terms of the bidistributions $\mathcal{G}_{\Theta}^{-}=\theta\left(t-t^{\prime}\right) \mathcal{G}_{\Theta}$ and $\mathcal{G}_{\Theta}^{+}=-\theta\left(t^{\prime}-t\right) \mathcal{G}_{\Theta}$, where $\mathcal{G}_{\Theta} \in \mathcal{D}^{\prime}(\stackrel{\circ}{N} \times \stackrel{\circ}{N})$ is such that, for all $f \in \mathcal{D}(\stackrel{\circ}{N})$

$$
\mathcal{G}_{\Theta}\left(f_{1}, f_{2}\right) \doteq \int_{\mathbb{R}^{2}} \mathrm{~d} t \mathrm{~d} t^{\prime}\left(f_{1}(t) \mid A_{\Theta}^{-\frac{1}{2}} \sin \left[A_{\Theta}^{\frac{1}{2}}\left(t-t^{\prime}\right)\right] f_{2}\left(t^{\prime}\right)\right),
$$

where $f(t) \in H^{2}(M)$ denotes the evaluation of $f$, regarded as an element of $C_{\mathrm{c}}^{\infty}\left(\mathbb{R}, H^{\infty}(M)\right)$ and $A_{\Theta}^{-\frac{1}{2}} \sin \left[A_{\Theta}^{\frac{1}{2}}\left(t-t^{\prime}\right)\right]$ is defined exploiting the functional calculus for $A_{\Theta}$. Moreover it holds that

$$
\mathrm{G}_{\Theta}^{ \pm}: \mathcal{D}(\stackrel{\circ}{N}) \rightarrow C^{\infty}\left(\mathbb{R}, H_{\Theta}^{\infty}(M)\right)
$$

where $H_{\Theta}^{\infty}(M) \doteq \bigcap_{k \geq 0} D\left(A_{\Theta}^{k}\right)$. In particular,

$$
\gamma_{1}\left(\mathrm{G}_{\Theta}^{ \pm} f\right)=\Theta \gamma_{0}\left(\mathrm{G}_{\Theta}^{ \pm} f\right) \quad \forall f \in C_{0}^{\infty}(\stackrel{\circ}{N}) .
$$

Proof. As starting point, we observe that, since per assumption $A_{\Theta}$ is bounded from below, the function $\sigma\left(A_{\Theta}\right) \ni \lambda \mapsto \lambda^{-1 / 2} \sin (\sqrt{\lambda} \tau)$ is smooth and bounded for all $\tau \in \mathbb{R}$. Hence, for any $f \in C_{0}^{\infty}(\stackrel{\circ}{N})(21)$ 
identifies an element $\mathrm{G}_{\Theta} f \in C^{\infty}\left(\mathbb{R}, D\left(A_{\Theta}^{1 / 2}\right)\right) \subset \mathcal{D}^{\prime}(\stackrel{\circ}{N})$. Moreover, for all $k \in \mathbb{N} \cup\{0\}$ and for all $t \in \mathbb{R}$, we have

$$
\left(1+A_{\Theta}\right)^{k}\left[\mathrm{G}_{\Theta} f\right](t)=\mathrm{G}_{\Theta}\left[\left(1+A_{\Theta}\right)^{k} f\right](t)=\mathrm{G}_{\Theta}\left[(1+A)^{k} f\right](t),
$$

which identifies an element of $L^{2}(M)$ since $(1+A)^{k} f \in \mathcal{D}(\stackrel{\circ}{N})$. Therefore $\left[\mathrm{G}_{\Theta} f\right](t)$ lies in $H_{\Theta}^{\infty}(M)$ and equation (22) follows. Notice that, since the hypothesis on $\beta$ guarantees that $A$ is a uniformly elliptic operator, $H_{\Theta}^{\infty}(M) \subset H^{\infty}(M)$.

The operator $\mathrm{G}_{\Theta}$ identifies unambiguously $\mathcal{G}_{\Theta} \in \mathcal{D}^{\prime}(\stackrel{\circ}{ } \times \stackrel{\circ}{N})$ satisfying per construction (20). In order to claim that $\mathcal{G}^{+} \doteq \theta\left(t-t^{\prime}\right) \mathcal{G}$ and $\mathcal{G}^{-}=-\theta\left(t^{\prime}-t\right) \mathcal{G}$ are the sought fundamental solutions we need to prove that $\operatorname{supp}\left(\mathcal{G}^{ \pm}(f)\right) \subseteq J^{\mp}(\operatorname{supp}(f))$. To this end it suffices to show that $\operatorname{supp}(\mathcal{G}(f)) \subseteq J(\operatorname{supp}(f))$, $J(\operatorname{supp}(f)) \doteq J^{-}(\operatorname{supp}(f)) \cup J^{+}(\operatorname{supp}(f))$.

This property is in turn a consequence of an energy estimate. Let $u(t) \doteq\left[\mathcal{G}_{\Theta}(f)\right](t)$ and let $K \subseteq M \simeq$ $\{0\} \times M$ be a compact set. We prove that, if $\left.u(0)\right|_{K}=\left.\dot{u}(0)\right|_{K}=0$, then $u$ vanishes on $U_{K} \doteq N \backslash J(M \backslash K)$. As a consequence $u(t)$ vanishes on $K_{t} \doteq M_{t} \cap U_{K}$ for all $t \in \mathbb{R}$, where $M_{t} \doteq\{t\} \times M$. To this end we introduce the positive energy functional

$$
E_{K}[u](t) \doteq \frac{1}{2}\left[C_{\infty}\|u(t)\|_{L^{2}\left(K_{t}\right)}^{2}+\|\dot{u}(t)\|_{L^{2}\left(K_{t}\right)}^{2}+\left\|\nabla_{\beta^{-1} g} u(t)\right\|_{L^{2}\left(K_{t}\right)}^{2}\right],
$$

where $\dot{u}(t)$ stands for $\frac{\mathrm{d}}{\mathrm{d} t} u(t)$ and where square integrability is defined still with respect to the metricinduced volume form, here left implicit. Moreover we set $C_{\infty} \doteq \sup _{U_{K}}|C|>0$ where $C:=A-\Delta_{\beta^{-1} g}$, see equation (18). A direct computation shows that

$$
\begin{aligned}
\frac{\mathrm{d}}{\mathrm{d} t} E_{K}[u](t) & =C_{\infty}(\dot{u}(t) \mid u(t))-\left(\dot{u}(t) \mid\left[\Delta_{\beta^{-1} g}+C\right] u(t)\right)+\left(\nabla_{\beta^{-1} g} \dot{u}(t) \mid \nabla_{\beta^{-1} g} u(t)\right) \\
& -\frac{1}{2}\left[C_{\infty}\left\|\gamma_{0, K_{t}} u(t)\right\|_{L^{2}\left(\partial K_{t}\right)}^{2}+\left\|\gamma_{0, K_{t}} \dot{u}(t)\right\|_{L^{2}\left(\partial K_{t}\right)}^{2}+\left\|\gamma_{1, K_{t}} u(t)\right\|_{L^{2}\left(\partial K_{t}\right)}^{2}\right],
\end{aligned}
$$

where $\left.\gamma_{0, K_{t}} u(t) \doteq u(t)\right|_{\partial K_{t}},\left.\gamma_{1, K_{t}} u(t) \doteq \nabla_{\beta^{-1} g} u(t)\right|_{\partial K_{t}}$ are the traces of $u(t), \nabla_{\beta^{-1} g} u(t)$ on $\partial K_{t}$, which are well-defined in view of the regularity of $u(t)$. The divergence theorem yields

$$
\begin{aligned}
\left(\nabla_{\beta^{-1} g} \dot{u}(t) \mid \nabla_{\beta^{-1} g} u(t)\right) & =\left(\gamma_{0, K_{t}} \dot{u}(t) \mid \gamma_{1, K_{t}} u(t)\right)_{L^{2}\left(\partial K_{t}\right)}+\left(\dot{u}(t) \mid \Delta_{\beta^{-1} g} u(t)\right) \\
& \leq \frac{1}{2}\left[\left\|\gamma_{0, K_{t}} \dot{u}(t)\right\|_{L^{2}\left(\partial K_{t}\right)}^{2}+\left\|\gamma_{1, K_{t}} u(t)\right\|_{L^{2}\left(\partial K_{t}\right)}^{2}\right]+\left(\dot{u}(t) \mid \Delta_{\beta^{-1} g} u(t)\right) .
\end{aligned}
$$

From this estimate we obtain a bound for the time derivative of $E_{K}[u](t)$, namely

$$
\frac{\mathrm{d}}{\mathrm{d} t} E_{K}[u](t) \leq C_{\infty}(\dot{u}(t) \mid u(t))-(\dot{u}(t) \mid C u(t)) \leq b E_{K}[u](t),
$$

where $b>0$. By Grönwall's inequality it follows that $E_{K}[u](t) \leq e^{b t} E_{K}[u](0)$ which vanishes because of our hypothesis on $u$. Since $E_{K}[u](t)$ is positive it follows that $u(t)=0$ vanishes on $K_{t}$ for all $t \in \mathbb{R}$. This proves that $\mathcal{G}$ enjoys the desired support property. In addition the energy estimate also entails the uniqueness of $\mathcal{G}$ and in turn of $\mathcal{G}^{ \pm}$. Suppose a second pair of retarded and advanced fundamental solutions exist, say $\mathcal{G}_{2}^{ \pm}$. Then, calling $\mathcal{G}_{2}=\mathcal{G}_{2}^{-}-\mathcal{G}_{2}^{+}$, it would hold that, for every $f \in \mathcal{D}(N), \mathcal{G}(f, \cdot)-\mathcal{G}_{2}(f, \cdot)$ is a solution of the D'Alembert wave equation with vanishing initial data. The energy estimate entails that this must be the zero solution, from which uniqueness descends.

Remark 31: One may wonder for which $\Theta$ the self-adjoint extension $A_{\Theta}$ is bounded from below. To the best of our knowledge this is an open problem. In Gru70 it was shown that, if the Dirichlet extension $A_{0}=\left.A^{*}\right|_{\text {ker } \gamma_{0}}$ has a finite lower bound $m\left(A_{0}\right)$ then $A_{\Theta}$ has a finite lower bound $m\left(A_{\Theta}\right)$ whenever that $\Theta$ has a lower bound such that $m(\Theta)>-m\left(A_{0}\right)$. Moreover, in this latter case it holds $m\left(A_{\Theta}\right)>m(\Theta) m\left(A_{0}\right)\left[m(\Theta)+m\left(A_{0}\right)\right]^{-1}$. 
Remark 32: Observe that, since the operator $A$ is per assumption uniformly elliptic, $H_{\Theta}^{\infty}(M) \subset H^{\infty}(M)$ where $H^{\infty}(M) \doteq \bigcap_{k \geq 0} H^{k}(M)$. In view of Remark[11, we can identify this space as the quotient between $H^{\infty}(\widehat{M})$ and the collection of those elements $v \in H^{\infty}(\widehat{M})$ such that $\left.v\right|_{M}=0$. Since $\widehat{M}$ is a metric complete Riemannian manifold, being of bounded geometry, it turns out that each element $v \in H^{\infty}(\widehat{M})$ admits a representative in $C^{\infty}(\widehat{M})$, see [Heb96, Ch. 3]. Consequently every $u \in H_{\Theta}^{\infty}(M)$ admits a representative lying in $C^{\infty}(M)$.

Remark 33: The previous analysis proves existence and uniqueness of the fundamental solutions for a wide class of boundary conditions. Yet it is important to mention that using the theory of boundary triples is not the only possible path which can be followed. In Pos18] self-adjoint extensions of the restriction of a given self-adjoint operator are classified via an efficient parametrization of their resolvent. In [ILP13, ILP14, a class of self-adjoint extensions of the Laplace-Beltrami operator on a smooth Riemannian manifold $M$ with smooth compact boundary $\partial M$ has been studied in the framework of quadratic forms. While the extension to a non-compact boundary has not been investigated yet, this method highlights that, among the collection of boundary conditions, a distinguished class is represented in terms of their interplay with the unitary representations of the isometry group of $\partial M$ acting on $L^{2}(\partial M)$.

Remark 34: We expect that a result similar in spirit to Theorem 30 can be derived also in the framework of [DS17, up to a suitable modification of the geometrical setting considered therein. This would allow to consider the wave operator with the insertion of an electromagnetic potential and with possibly low regularity of both the metric and the electromagnetic potential in the sense of [DS17]. We are currently investigating this topic.

To conclude the analysis of the structural properties of the fundamental solutions, we extend to the case at hand a result which, in the case of globally hyperbolic spacetimes, was proven in BGP07, Th. 3.4.7]. The goal is to prove that the advanced and retarded Green operators allow for a complete characterization of the space of smooth solutions of the D'Alembert wave equation. In view of equations (16) and (17) we can still work with the operator $\partial_{t}^{2}+A$. To this end, first of all we need to enlarge the domain of definition of the operators $\mathrm{G}_{\Theta}^{ \pm}$defined in Theorem 30 .

Definition 35: Under the same hypotheses of Theorem 30, let $H_{\Theta}^{\infty}(M) \doteq \bigcap_{k \geq 1} D\left(A_{\Theta}^{k}\right)$. Hence, motivated by the nomenclature in [Bar15] we call:

1. $C_{\mathrm{fc}}^{\infty}\left(\mathbb{R}, H_{\Theta}^{\infty}(M)\right)$ the space of future compact, $H_{\Theta}^{\infty}$-valued smooth functions, that is the collection of $f \in C^{\infty}\left(\mathbb{R}, H_{\Theta}^{\infty}(M)\right)$ for which $J_{\mathbb{R} \times{ }_{\beta} M}^{+}(x) \cap \operatorname{supp}(f)$ is compact or empty for all $x \in \mathbb{R} \times{ }_{\beta} M$, where $J_{\mathbb{R} \times{ }_{\beta} M}^{+}$stands for the causal future in $\mathbb{R} \times_{\beta} M$ endowed with the metric (15),

2. $C_{\mathrm{pc}}^{\infty}\left(\mathbb{R}, H_{\Theta}^{\infty}(M)\right)$ the space of past compact $H_{\Theta}^{\infty}(M)$-valued smooth functions, that is the collection of $f \in C^{\infty}\left(\mathbb{R}, H_{\Theta}^{\infty}(M)\right)$ for which $J_{\mathbb{R} \times_{\beta} M}^{-}(x) \cap \operatorname{supp}(f)$ is compact or empty for all $x \in \mathbb{R} \times{ }_{\beta} M$, where $J_{\mathbb{R} \times{ }_{\beta} M}^{-}$stands for the causal past in $\mathbb{R} \times_{\beta} M$ endowed with the metric (15),

3. $C_{\mathrm{tc}}^{\infty}\left(\mathbb{R}, H_{\Theta}^{\infty}(M)\right) \doteq C_{\mathrm{pc}}^{\infty}\left(\mathbb{R}, H_{\Theta}^{\infty}(M)\right) \cap C_{\mathrm{fc}}^{\infty}\left(\mathbb{R}, H_{\Theta}^{\infty}(M)\right)$ the collection of timelike compact $H_{\Theta}^{\infty}(M)$ valued smooth functions.

By combining Theorem 30 and the results of [Bar15], it descends that $\mathrm{G}_{\Theta}^{ \pm}$extend respectively to linear maps

$$
\mathrm{G}_{\Theta}^{+}: C_{\mathrm{fc}}^{\infty}\left(\mathbb{R}, H_{\Theta}^{\infty}(M)\right) \rightarrow C^{\infty}\left(\mathbb{R}, H_{\Theta}^{\infty}(M)\right), \quad \mathrm{G}_{\Theta}^{-}: C_{\mathrm{pc}}^{\infty}\left(\mathbb{R}, H_{\Theta}^{\infty}(M)\right) \rightarrow C^{\infty}\left(\mathbb{R}, H_{\Theta}^{\infty}(M)\right),
$$

which preserve the properties $\square_{\Theta} \circ \mathrm{G}_{\Theta}^{ \pm}=\mathrm{G}_{\Theta}^{ \pm} \circ \square_{\Theta}=\mathrm{id}$ and $\operatorname{supp}\left(\mathrm{G}^{ \pm} f\right) \subseteq J^{\mp}(\operatorname{supp}(f))$. 
Proposition 36: Under the hypotheses of Theorem 30, let $\square_{\Theta} \doteq \partial_{t}^{2}+A_{\Theta}$. Then the following is an exact sequence of linear maps:

$$
0 \rightarrow C_{\mathrm{tc}}^{\infty}\left(\mathbb{R}, H_{\Theta}^{\infty}(M)\right) \stackrel{\square_{\Theta}}{\longrightarrow} C_{\mathrm{tc}}^{\infty}\left(\mathbb{R}, H_{\Theta}^{\infty}(M)\right) \stackrel{\mathrm{G}_{\Theta}}{\longrightarrow} C^{\infty}\left(\mathbb{R}, H_{\Theta}^{\infty}(M)\right) \stackrel{\square_{\Theta}}{\longrightarrow} C^{\infty}\left(\mathbb{R}, H_{\Theta}^{\infty}(M)\right) \rightarrow 0 .
$$

Proof. The proof follows the same steps of [BGP07, Th. 3.4.7]. Let $f \in C_{\mathrm{tc}}^{\infty}\left(\mathbb{R}, H_{\Theta}^{\infty}(M)\right)$ be such that $\square_{\Theta} f=0$. Hence $f=\mathrm{G}_{\Theta}^{+} \square_{\Theta} f=0$, which shows the injectivity of the first arrow ruled by $\square_{\Theta}$.

For all $f \in C_{\mathrm{tc}}^{\infty}\left(\mathbb{R}, H_{\Theta}^{\infty}(M)\right)$ it holds from Definition 27 that $\square_{\Theta} \mathrm{G}_{\Theta} f=\mathrm{G}_{\Theta} \square_{\Theta} f=0$. Therefore $\square_{\Theta} C_{\mathrm{tc}}^{\infty}\left(\mathbb{R}, H_{\Theta}^{\infty}(M)\right) \subseteq \operatorname{ker} \mathrm{G}_{\Theta}$. Let now $f \in C_{\mathrm{tc}}^{\infty}\left(\mathbb{R}, H_{\Theta}^{\infty}(M)\right)$ be such that $\mathrm{G}_{\Theta} f=0$. Then $\psi=\mathrm{G}_{\Theta}^{+} f=-\mathrm{G}^{-} f$ belongs to $C_{\mathrm{tc}}^{\infty}\left(\mathbb{R}, H_{\Theta}^{\infty}(M)\right)$ and $\square_{\Theta} \psi=f$, which entails $f \in \square_{\Theta} C_{\Theta}^{\infty}\left(\mathbb{R}, H_{\Theta}^{\infty}(M)\right)$. Hence $\operatorname{Im}\left(\square_{\Theta}\right)=\operatorname{ker} G_{\Theta}$ which prove the exactness of the second and of third arrow. We now show that $\mathrm{G}_{\Theta}\left[C_{\mathrm{tc}}^{\infty}\left(\mathbb{R}, H_{\Theta}^{\infty}(M)\right)\right]=\operatorname{ker}\left[\left.\square_{\Theta}\right|_{C^{\infty}\left(\mathbb{R}, H_{\Theta}^{\infty}(M)\right)}\right]$. The inclusion $\subseteq$ follows from $\square_{\Theta} \mathrm{G}_{\Theta} C_{\mathrm{tc}}^{\infty}\left(\mathbb{R}, H_{\Theta}^{\infty}(M)\right)=0$. Let $f \in C^{\infty}\left(\mathbb{R}, H_{\Theta}^{\infty}(M)\right)$ be such that $\square_{\Theta} f=0$. Let us consider its (non-unique) decomposition as $f=f_{+}+f_{-}$with $f_{ \pm} \in C^{\infty}\left(\mathbb{R}, H_{\Theta}^{\infty}(M)\right)$ where $f_{+}$(resp. $f_{-}$) is future (resp. past) compact. It follows that $\square_{\Theta} f_{-} \in C_{\mathrm{pc}}^{\infty}\left(\mathbb{R}, H_{\Theta}^{\infty}(M)\right) \subset C^{\infty}\left(\mathbb{R}, H_{\Theta}^{\infty}(M)\right)$ and from the support properties of $\mathrm{G}_{\Theta}^{ \pm}$combined with $\square_{\Theta} f_{+}=-\square_{\Theta} f_{-}$,

$$
\mathrm{G}_{\Theta} \square_{\Theta} f_{-}=\mathrm{G}_{\Theta}^{-} \square_{\Theta} f_{-}+\mathrm{G}_{\Theta}^{+} \square_{\Theta} f_{+}=f_{+}+f_{-}=f,
$$

from which it descends the exactness of the third and forth arrows. Finally, the last arrow entails instead that, for every $h \in C^{\infty}\left(\mathbb{R}, H_{\Theta}^{\infty}(M)\right)$ there exists $f \in C^{\infty}\left(\mathbb{R}, H_{\Theta}^{\infty}(M)\right)$ such that $\square_{\Theta} f=h$. Similarly to the proof of the previous points, it suffices to split $h=h_{+}+h_{-}$where $h_{+} \in C_{\mathrm{fc}}^{\infty}\left(\mathbb{R}, H_{\Theta}^{\infty}(M)\right)$ while $h_{-} \in$ $C_{\mathrm{pc}}^{\infty}\left(\mathbb{R}, H_{\Theta}^{\infty}(M)\right)$. As a consequence of the properties of $\mathrm{G}_{\Theta}^{ \pm}$, the sought function is $f=\mathrm{G}^{+} h_{+}+\mathrm{G}^{-} h_{-}$.

Remark 37: Observe that another, equivalent way of reading the sequence (23) of Proposition 36 is that the causal propagator $\mathrm{G}_{\Theta} \doteq \mathrm{G}_{\Theta}^{-}-\mathrm{G}_{\Theta}^{+}$induces an isomorphism between the quotient of vector spaces $\frac{C_{\mathrm{t}}^{\infty}\left(\mathbb{R}, H_{\Theta}^{\infty}(M)\right)}{\square\left[C_{\mathrm{tc}}^{\infty}\left(\mathbb{R}, H_{\Theta}^{\infty}(M)\right)\right]}$ and $\mathcal{S}_{\Theta}\left(\mathbb{R} \times_{\beta} N\right) \doteq\left\{u \in C^{\infty}\left(\mathbb{R} ; H_{\Theta}^{\infty}(M)\right) \mid \square_{\Theta} u=0\right\}$.

Example 38: To better illustrate the above analysis we discuss in detail an explicit example often used in the literature. Most notably we consider a static Lorentzian spacetime with boundary $N=M \times \mathbb{R}$ with warping factor $\beta=1$. The underlying manifold $(M, g)$ is assumed to be $\mathbb{R}_{+} \times \mathbb{R}^{n}, n \geq 0$, endowed with the standard Euclidean metric. Let $\left(\gamma_{0}, \gamma_{1}, L^{2}\left(\mathbb{R}^{n}\right)\right)$ be the boundary triple as per Proposition 24] for $n>1$ while, for $n=0$ the boundary Hilbert space is $\mathbb{C}$. The Laplace-Beltrami operator associated to $(M, g)$ reads in Cartesian coordinates

$$
\Delta_{g}=-\partial_{x_{1}}^{2}-\sum_{i=2}^{n+1} \partial_{x_{i}}^{2}
$$

and we indicate with $\Delta_{\Theta}$ the self-adjoint extension induced by $\Theta$ according to Theorem 23, If $n=0$ $\Delta$ becomes simply $-\partial_{x_{1}}^{2}$. This is a special case, for which the following discussion is not necessary. In order to apply Theorem 30 the first step consists of checking that $\Delta_{\Theta}$ is bounded from below. Taking the Fourier transform $\mathcal{F}_{\mathbb{R}^{n}}$ in all variables barring $x_{1}$ and using the notational shortcut $k^{2} \doteq \sum_{i=2}^{n+1} k_{i}^{2}$ this question reduces to the study of the spectral property of

$$
\widetilde{\Delta} \doteq\left(1 \otimes \mathcal{F}_{\mathbb{R}^{n}}\right)^{-1} \circ \Delta_{g} \circ\left(1 \otimes \mathcal{F}_{\mathbb{R}^{n}}\right)=-\partial_{x_{1}}^{2}+k^{2} .
$$

defined on the domain (the tilde symbol indicating that the domains refer to the operator $\widetilde{\Delta}$ )

$$
\widetilde{H}_{0}^{2}\left(\mathbb{R}_{+} \times \mathbb{R}^{n}\right)=\left\{\psi \in L^{2}\left(\mathbb{R}_{+} \times \mathbb{R}^{n}\right) \mid \partial_{x_{1}}^{2} \psi, k^{2} \psi \in L^{2}\left(\mathbb{R}_{+} \times \mathbb{R}^{n}\right), \quad \gamma_{0}(\psi)=\gamma_{1}(\psi)=0\right\} .
$$


The adjoint is defined on

$$
\widetilde{H}^{2}\left(\mathbb{R}_{+} \times \mathbb{R}^{n}\right)=\left\{\psi \in L^{2}\left(\mathbb{R}_{+} \times \mathbb{R}^{n}\right) \mid \partial_{x_{1}}^{2} \psi, k^{2} \psi \in L^{2}\left(\mathbb{R}_{+} \times \mathbb{R}^{n}\right)\right\}, \quad\left[\widetilde{\Delta}_{h}\right]^{*} \psi=\widetilde{\Delta}_{h} \psi
$$

The deficiency indexes of $\widetilde{\Delta}$ are equal, in particular $\operatorname{ker}(\widetilde{\Delta} \pm i) \simeq L^{2}\left(\mathbb{R}^{n}\right)$. According to Proposition 19 any self-adjoint extension of $\widetilde{\Delta}$ can be obtained from a self-adjoint operator $\widetilde{\Theta}$ on $L^{2}(\mathbb{R})$ as

$$
D\left(\widetilde{\Delta}_{\widetilde{\Theta}}\right) \doteq\left\{\psi \in \widetilde{H}^{2}\left(\mathbb{R}_{+} \times \mathbb{R}^{n}\right) \mid \gamma_{0}(\psi) \in D(\widetilde{\Theta}), \quad \gamma_{1}(\psi)=\widetilde{\Theta} \gamma_{0}(\psi)\right\},
$$

where, for smooth $\psi,\left(\gamma_{0} \psi\right)(y)=\psi(0, y)$ while $\left(\gamma_{1} \psi\right)(y)=\partial_{x} \psi(0, y)$. Notice that, since $1 \otimes \mathcal{F}_{\mathbb{R}^{n}}: L^{2}\left(\mathbb{R}_{+} \times\right.$ $\left.\mathbb{R}^{n}\right) \rightarrow L^{2}\left(\mathbb{R}_{+} \times \mathbb{R}^{n}\right)$ is a unitary operator, each self adjoint extension $\widetilde{\Delta}_{\widetilde{\Theta}}$ corresponds to a self adjoint counterpart $\Delta_{\Theta}$ where $\widetilde{\Theta} \doteq\left(1 \otimes \mathcal{F}_{\mathbb{R}^{n}}\right)^{-1} \circ \Theta \circ\left(1 \otimes \mathcal{F}_{\mathbb{R}^{n}}\right)$.

In order to evaluate the spectrum of $\widetilde{\Delta}_{\widetilde{\Theta}}$, first we focus on the Dirichlet self-adjoint extension

$$
D\left(\widetilde{\Delta}_{\infty}\right) \doteq\left\{\psi \in \widetilde{H}^{2}\left(\mathbb{R}_{+} \times \mathbb{R}^{n}\right) \mid \gamma_{0}(\psi)=0\right\} .
$$

For each $\psi \in D\left(\widetilde{\Delta}_{\infty}\right)$, ext $[\psi]\left(x_{1}, k\right)=\theta\left(x_{1}\right) \psi\left(x_{1}, k\right)-\theta\left(-x_{1}\right) \psi\left(-x_{1}, k\right)$ identifies an element in $\widetilde{H}^{2}\left(\mathbb{R}^{n+1}\right)=$ $\left(1 \otimes \mathcal{F}_{\mathbb{R}^{n+1}}\right) H^{2}\left(\mathbb{R}^{n+1}\right), \theta$ being the Heaviside distribution. The sine transform along the $x_{1}$-variable yields

$$
\widetilde{\Delta}_{\infty} \psi\left(x_{1}, k\right)=\frac{2}{\pi} \int_{\mathbb{R}_{+}} \mathrm{d} \xi\left(\xi^{2}+k^{2}\right) \sin \left(\xi x_{1}\right)\left[\int_{\mathbb{R}_{+}} \mathrm{d} y_{1} \sin \left(\xi y_{1}\right) \psi\left(y_{1}, k\right)\right],
$$

from which we read that $\widetilde{\Delta}_{\infty}$ coincides with the multiplication operator by $\xi^{2}+k^{2}$, which entails, in turn, $\sigma\left(\widetilde{\Delta}_{\infty}\right)=\sigma\left(\Delta_{\infty}\right)=(0,+\infty)$.

The remaining contribution to the spectrum of $\widetilde{\Delta}_{\widetilde{\Theta}}$ can be studied via Theorem 23. In particular the Weyl function associated with the boundary triple $\left(L^{2}\left(\mathbb{R}^{n}\right), \gamma_{0}, \gamma_{1}\right)$ is,

$$
[M(\lambda) g](k)=-\sqrt{k^{2}-\lambda} g(k) \quad \text { for } \lambda \in \rho\left(\widetilde{\Delta}_{\infty}\right) \cap \mathbb{R}=(-\infty, 0) .
$$

Still applying Theorem 23 we obtain that $\lambda \in \sigma\left(\widetilde{\Delta}_{\widetilde{\Theta}}\right)$ if and only if $0 \in \sigma(\widetilde{\Theta}-M(\lambda))=\sigma\left(\widetilde{\Theta}+\sqrt{k^{2}-\lambda}\right)$. At this stage we specialize to a specific scenario: Robin boundary conditions. In other words $\Theta=\alpha \mathbb{I}, \mathbb{I}$ being the identity operator on $L^{2}\left(\mathbb{R}^{n}\right)$, while $\alpha \in \mathbb{R}$. Hence also $\widetilde{\Theta}=\alpha \mathbb{I}$ and it descends

$$
\sigma\left(\widetilde{\Theta}+\sqrt{k^{2}-\lambda}\right)=\left\{\alpha+\sqrt{k^{2}-\lambda} \mid k \in \mathbb{R}\right\} .
$$

Thus if $\alpha \geq 0,0 \notin \sigma(\Theta-M(\lambda))$, while, if $\alpha<0$, all negative values of $\lambda$ greater that $-\alpha^{2}$ are allowed. Hence $\widetilde{\Delta}_{\alpha \mathbb{I}}$ is bounded from below for all $\alpha \in \mathbb{R}$ with $\sigma\left(\widetilde{\Delta}_{\alpha \mathbb{I}}\right)=\left(-\alpha^{2},+\infty\right)$ for $\alpha<0$, while $\sigma\left(\widetilde{\Delta}_{\alpha \mathbb{I}}\right)=(0,+\infty)$ for $\alpha \geq 0$. A special scenario occurs if we consider $n=0$, since the Weyl function reads $M(\lambda)=\sqrt{-\lambda}$. In this case, if $\alpha<0$, the only admissible, negative value for $\lambda$ is $-\alpha^{2}$ and thus all conditions of Theorem 23 are met. We observe that this result is consistent with that of [DF17] for the study of a massless, conformally coupled real, scalar field on the Poincaré patch of AdS spacetime.

We can thus write the causal propagator $\widetilde{\mathrm{G}}_{\alpha \mathbb{I}}$ for $\partial_{t}^{2}+\widetilde{\Delta}$ explicitly. This is tantamount to computing $\mathrm{G}_{\alpha \mathbb{I}}$ up to the Fourier transform in all spatial variables barring $x_{1}$. We have

$$
\begin{aligned}
{\left[\widetilde{\mathrm{G}}_{\alpha \mathbb{I}} f\right]\left(t, x_{1}, k\right) } & =\int_{\mathbb{R}} \int_{\mathbb{R}_{+}} \sin \left[\left(\xi^{2}+k^{2}\right)^{\frac{1}{2}}(t-s)\right]\left(\xi^{2}+k^{2}\right)^{-\frac{1}{2}} \psi\left(x_{1}, \xi\right)\left[\int_{\mathbb{R}_{+}} \psi\left(y_{1}, \xi\right) f\left(s, y_{1}, k\right) \mathrm{d} y_{1}\right] \mathrm{d} \xi \mathrm{d} s \\
& -\int_{\mathbb{R}} \sinh \left[\left(\alpha^{2}-k^{2}\right)^{\frac{1}{2}}(t-s)\right]\left(\alpha^{2}-k^{2}\right)^{-\frac{1}{2}} 1_{(-\alpha, \alpha)}(k) e_{\alpha}\left(x_{1}\right)\left[\int_{\mathbb{R}_{+}} e_{\alpha}\left(y_{1}\right) f\left(s, y_{1}, k\right) \mathrm{d} y_{1}\right] \mathrm{d} s
\end{aligned}
$$


where we defined

$$
\psi\left(x_{1}, \xi\right) \doteq\left(\xi^{2}+\alpha^{2}\right)^{-\frac{1}{2}}\left[\xi \cos \left(\xi x_{1}\right)+\alpha \sin \left(\xi x_{1}\right)\right] \quad e_{\alpha}(x) \doteq(2 \alpha)^{\frac{1}{2}} e^{-\alpha x} .
$$

Another relevant choice for $\Theta$ is a differential operator. This may model a Riemannian version of the well-known Wentzell boundary conditions (which will be treated in the next section). In particular, in this latter case the operator $\widetilde{\Theta}$ acts as $[\widetilde{\Theta} f](k)=p_{\Theta}(k) f(k)$ where $p_{\Theta}$ is the symbol associated with $\Theta$ via Fourier transform. Thus the condition for $\lambda<0$ to be in the spectrum of $\Delta_{\Theta}$ is that $0 \in$ $\left\{p_{\Theta}(k)-\sqrt{k^{2}-\lambda} \quad k \in \mathbb{R}\right\}$.

\subsection{Dynamical boundary conditions}

Theorem 30 and the analysis of the previous section were tied to the construction of the advanced and retarded Green's operators $\mathrm{G}_{\Theta}^{ \pm}$in terms of boundary conditions ascribed to a choice both of boundary triple and of a self-adjoint operator $\Theta$ on the boundary Hilbert space $L^{2}(\partial M)$. From the Lorentzian viewpoint, this scenario can be interpreted as assigning via $\Theta$ a time-independent boundary condition.

Nonetheless, in many models and applications, this is not sufficient since one wishes to relax the hypothesis of time-independence, see e.g. [Fel57, Z18, for a specific example. We show how it is possible to extend our previous analysis to encompass also most of these scenarios.

In the rest of the section, we consider $(N, h)$, a generic Lorentzian, static spacetime, with timelike boundary where $h$ is given by equation (15). We assume that the warping factor $\beta$ is chosen in such a way that, writing the D'Alembert wave operator as $\square=\partial_{t}^{2}+A$ with $A$ defined as per equation (18), $A$ is a uniformly elliptic operator on $L^{2}(M)$.

In addition, let $\left(\gamma_{0}, \gamma_{1}, L^{2}(\partial M)\right)$ be the boundary triple associated to $A^{*}$ as per (11). With $\left(\widetilde{\gamma}_{0}, \widetilde{\gamma}_{1}\right)$ we denote the natural extension of $\left(\gamma_{0}, \gamma_{1}\right)$ to $C^{\infty}\left(\mathbb{R} ; D\left(A^{*}\right)\right)$, while the boundary condition is implemented by restricting to the subspace of $C^{\infty}\left(\mathbb{R} ; D\left(A^{*}\right)\right)$ whose elements $u$ satisfy

$$
\widetilde{\gamma}_{1} u=\left(\partial_{t}^{2}+\widetilde{\Theta}\right) \widetilde{\gamma}_{0} u,
$$

where $\widetilde{\Theta}$ is the natural extension to $C^{\infty}(\mathbb{R}, D(\Theta))$ of a self-adjoint operator $\Theta: D(\Theta) \subseteq L^{2}(\partial M) \rightarrow$ $L^{2}(\partial M)$. Observe that, being the background static, $\partial_{t}^{2} \widetilde{\gamma}_{0}=\widetilde{\gamma}_{0} \partial_{t}^{2}$.

In order to address the problem of existence and uniqueness of the advanced and retarded Green's operators for the system at hand we make use of a technique which extends the one used in [Fel57, Z18. It consists of rewriting the wave equation (20) with boundary conditions prescribed via (24) in terms of the following equivalent system: Let $\widehat{u}=(u, v) \in C^{\infty}\left(\mathbb{R}, D\left(A^{*}\right)\right) \times C^{\infty}(\mathbb{R}, D(\Theta))$

$$
\widehat{\mathrm{P}} \widehat{u} \doteq \partial_{t}^{2} \widehat{u}+\widehat{A}_{\Theta} \widehat{u}=0, \quad \widehat{A}_{\Theta}=\left(\begin{array}{cc}
\widetilde{A}^{*} & 0 \\
-\widetilde{\gamma}_{1} & \widetilde{\Theta}
\end{array}\right), \quad \widetilde{\gamma}_{0}(u)=v,
$$

where $\widetilde{A}^{*}$ indicates the natural extension of $A^{*}$ to $C^{\infty}\left(\mathbb{R} ; D\left(A^{*}\right)\right)$.

Observe that in (25) we have introduced the auxiliary operator $\widehat{A}_{\Theta}$ which acts on $C^{\infty}\left(\mathbb{R} ; D\left(\widehat{A}_{\Theta}\right)\right)$, where $D\left(\widehat{A}_{\Theta}\right)$ is the subset of $\widehat{\mathrm{H}} \doteq \mathrm{H} \oplus \mathrm{h}=L^{2}(M) \oplus L^{2}(\partial M)$, the Hilbert space with scalar product

$$
\left(\widehat{\phi}_{1} \mid \widehat{\phi}_{2}\right)_{\widehat{\mathrm{H}}}=\left(\phi_{1} \mid \phi_{2}\right)_{\mathrm{H}}+\left(\varphi_{1} \mid \varphi_{2}\right)_{\mathrm{h}}, \quad \widehat{\phi}_{i}=\left(\phi_{i}, \varphi_{i}\right), i=1,2,
$$

such that

$$
D\left(\widehat{A}_{\Theta}\right) \doteq\left\{\widehat{\phi}=(\phi, \varphi) \in \widehat{\mathrm{H}} \mid \phi \in H^{2}(M), \varphi \in D(\Theta), \gamma_{0} \phi=\varphi\right\}, \quad \widehat{A}_{\Theta} \widehat{\phi}=\left(\begin{array}{l}
A^{*} \phi \\
\Theta \varphi-\gamma_{1} \phi
\end{array}\right) .
$$


The Hilbert space structure on $\widehat{H}$ is defined by

$$
\left(\widehat{\phi}_{1} \mid \widehat{\phi}_{2}\right)_{\widehat{\mathrm{H}}}=\left(\phi_{1} \mid \phi_{2}\right)_{\mathrm{H}}+\left(\varphi_{1} \mid \varphi_{2}\right)_{\mathrm{h}}, \quad \widehat{\phi}_{i}=\left(\phi_{i}, \varphi_{i}\right), i=1,2 .
$$

We recall that $\Theta: D(\Theta) \subseteq \mathrm{h} \rightarrow \mathrm{h}$ is a self-adjoint operator on $\mathrm{h}$ and that $\left(\mathrm{h}, \gamma_{0}, \gamma_{1}\right)$ is a boundary triple for $A^{*}$. We can prove a relevant property of $\widehat{A}_{\Theta}$.

Proposition 39: Let $\left(\mathrm{h}, \gamma_{0}, \gamma_{1}\right)$ be the boundary triple associated with $A^{*}$ as per Proposition 24. Let $\Theta: D(\Theta) \subseteq \mathrm{h} \rightarrow \mathrm{h}$ be a self adjoint operator. Then the operator $\widehat{A}_{\Theta}: D\left(\widehat{A}_{\Theta}\right) \subseteq \widehat{\mathrm{H}} \rightarrow \widehat{\mathrm{H}}$, defined as per equation (26), is self-adjoint.

Proof. Since $\gamma_{0}$ is a surjective map, while $D(\Theta)$ is dense in $\mathrm{h}$ and $H_{0}^{1}(M)=\operatorname{ker} \gamma_{0}, D\left(\widehat{A}_{\Theta}\right)$ is dense in $\widehat{\mathrm{H}}$. On account of equation (4) it holds $\left(\widehat{A}_{\Theta} \widehat{\phi} \mid \widehat{\psi}\right)=\left(\widehat{\phi} \mid \widehat{A}_{\Theta} \widehat{\psi}\right)$ for all $\widehat{\phi}, \widehat{\psi} \in D\left(\widehat{A}_{\Theta}\right)$, that is, $\widehat{A}_{\Theta}$ is a symmetric operator. We prove that $D\left(\widehat{A}_{\Theta}^{*}\right) \subseteq D\left(\widehat{A}_{\Theta}\right)$. Let $\widehat{\phi} \in D\left(\widehat{A}_{\Theta}^{*}\right)$. The map $D\left(\widehat{A}_{\Theta}\right) \ni \widehat{\rho} \mapsto\left(\widehat{\phi} \mid \widehat{A}_{\Theta} \widehat{\rho}\right) \in \mathbb{C}$ is bounded. A direct inspection shows that, if $\widehat{\phi}=(\phi, \varphi)$ and $\widehat{\rho}=(\rho, \varrho)$,

$$
\left(\widehat{\phi} \mid \widehat{A}_{\Theta} \widehat{\rho}\right)_{\widehat{\mathrm{H}}}=\left(\phi \mid A^{*} \rho\right)_{\mathrm{H}}+\left(\varphi \mid \Theta \varrho-\gamma_{1} \rho\right)_{\mathrm{h}} .
$$

If $\rho \in \operatorname{ker} \gamma_{1} \cap \operatorname{ker} \gamma_{0}=H_{0}^{2}(M)$, the last term on the right hand side of equation (27) vanishes and the boundedness of the left hand side implies that $\phi \in D\left(A^{*}\right)=H^{2}(M)$. By applying equation (4) we find

$$
\left(\widehat{A}_{\Theta}^{*} \widehat{\phi} \mid \widehat{\rho}\right)_{\widehat{H}}=\left(\widehat{\phi} \mid \widehat{A}_{\Theta} \widehat{\rho}\right)_{\hat{\mathrm{H}}}=(\phi \mid A \rho)_{\mathrm{H}}=\left(A^{*} \phi \mid \rho\right)_{\mathrm{H}},
$$

where in the last equality we used equation (44). This shows that $\operatorname{pr}_{1} \widehat{A}_{\Theta}^{*} \widehat{\phi}=A^{*} \phi$ where $\operatorname{pr}_{1}: \widehat{\mathrm{H}} \rightarrow L^{2}(M)$ is the projection on the first component.

Let us now consider $\widehat{\rho} \in D\left(\widehat{A}_{\Theta}\right)$. Since $\phi \in H^{2}(M)$, we can combine equations (27) and equation (4) for the boundary triple $\left(\mathrm{h}, \gamma_{0}, \gamma_{1}\right)$ to obtain

$$
\left(\widehat{\phi} \mid \widehat{A}_{\Theta} \widehat{\rho}\right)_{\widehat{\mathrm{H}}}=\left(A^{*} \phi \mid \rho\right)_{\mathrm{H}}+\left(\gamma_{0} \phi \mid \gamma_{1} \rho\right)_{\mathrm{h}}-\left(\gamma_{1} \phi \mid \varrho\right)_{\mathrm{h}}+(\varphi \mid \Theta \varrho)_{\mathrm{h}}-\left(\varphi \mid \gamma_{1} \rho\right)_{\mathrm{h}} .
$$

In particular, the boundedness condition on $\widehat{\rho} \mapsto\left(\widehat{\phi} \mid \widehat{A}_{\Theta} \widehat{\rho}\right)$ implies that $\varphi \in D\left(\Theta^{*}\right)=D(\Theta)$. Therefore equation (29) simplifies further and we conclude that

$$
\left(\widehat{A}_{\Theta}^{*} \widehat{\phi} \mid \widehat{\rho}\right)_{\widehat{H}}-\left(\widehat{A}_{\Theta} \widehat{\phi} \mid \widehat{\rho}\right)_{\widehat{\mathrm{H}}}=\left(\gamma_{0} \phi-\varphi \mid \gamma_{1} \rho\right)_{\mathrm{h}} .
$$

If $\rho \in \operatorname{ker} \gamma_{0}$, the left hand side vanishes - we recall that $\operatorname{pr}_{1} \widehat{A}_{\Theta}^{*} \widehat{\phi}=A^{*} \phi$. Since $\left(\gamma_{0}, \gamma_{1}\right)$ is a surjective map, it follows that $\gamma_{0}(\phi)=\varphi$, that is, $\widehat{\phi} \in D\left(\widehat{A}_{\Theta}\right)$.

Remark 40: Proposition [39 can be easily generalized to the case of an arbitrary boundary triple (h, $\left.\gamma_{0}, \gamma_{1}\right)$ for the adjoint $S^{*}$ of a densely defined symmetric operator $S$ on an Hilbert space $\mathrm{H}$ 过. In this latter case, $\Theta$ is a densely defined self-adjoint operator on $\mathrm{h}$, while the operator $\widehat{S}_{\Theta}$ is defined on $D\left(\widehat{S}_{\Theta}\right) \subset \widehat{\mathrm{H}} \doteq \mathrm{H} \oplus \mathrm{h}$, in particular,

$$
D\left(\widehat{S}_{\Theta}\right) \doteq\left\{\widehat{\phi} \in \widehat{\mathrm{H}} \mid \phi \in D\left(S^{*}\right), \varphi \in D(\Theta), \gamma_{0} \phi=\varphi\right\},
$$

where $\widehat{\phi}=(\phi, \varphi)$.

\footnotetext{
${ }^{4}$ For the sake of completeness, one should assume that $\gamma_{0}$ is not closable with respect to the norm topology of $\mathrm{H}-$ in order to ensure that $D\left(\widehat{A}_{\Theta}\right)$ is dense in $\widehat{\mathrm{H}}$ : This is the case in all applications.
} 
Remark 41: The spectral properties of $\widehat{A}_{\Theta}$ can be linked to elliptic boundary value problems with spectral-dependent boundary conditions, see for example [Beh10] and [BL12]. Indeed, $\lambda \in \rho\left(\widehat{A}_{\Theta}\right)$ implies that $\lambda \in \rho\left(A_{\Theta-\lambda}\right)$, where $A_{\Theta-\lambda}$ denotes the self-adjoint extension of $A$ defined via Proposition [19.

We can now formulate the counterpart of Theorem 30 for the case at hand. Since the proof is the same mutatis mutandis, we will omit it:

Theorem 42: Let $\left(\mathrm{h}, \gamma_{0}, \gamma_{1}\right)$ be the boundary triple associated with $A^{*}$ as per Proposition 24, Let $\Theta: D(\Theta) \subseteq \mathrm{h} \rightarrow \mathrm{h}$ be a self-adjoint operator and let assume that the spectrum of the self-adjoint operator $\widehat{A}_{\Theta}$ defined in Proposition 39 is bounded from below. Finally let $\operatorname{pr}_{1}: \mathrm{H} \rightarrow L^{2}(M)$ be the projection to the first component of $\widehat{\mathrm{H}}$ and let ext: $H^{2}(M) \rightarrow \widehat{\mathrm{H}}_{\gamma_{0}}$ be the extension map $\phi \mapsto \widehat{\phi}_{\text {ext }} \doteq\left(\phi, \gamma_{0} \phi\right)$. For $f \in C^{\infty}(\stackrel{N}{)})$, let

$$
\left[\mathrm{G}_{\Theta} f\right](t) \doteq \operatorname{pr}_{1}\left[\int_{\mathbb{R}} \widehat{A}_{\Theta}^{-\frac{1}{2}} \sin \left[\widehat{A}_{\Theta}^{\frac{1}{2}}\left(t-t^{\prime}\right)\right] \widehat{f}_{\mathrm{ext}}\left(t^{\prime}\right) \mathrm{d} t^{\prime}\right] .
$$

Then $\left(\mathrm{G}_{\Theta}^{+}, \mathrm{G}_{\Theta}^{-}\right)$where $\mathrm{G}^{+}=\theta\left(t-t^{\prime}\right) \mathrm{G}$ and $\mathrm{G}^{-}=-\theta\left(t^{\prime}-t\right) \mathrm{G}$ is a pair of advanced and retarded Green's operators for $\mathrm{P}$ as per Definition 27) subjected to the boundary conditions (24).

Example 43: With reference to the same geometric setting as in Example 38 we apply the previous analysis, Theorem 42 in particular, to the dynamical boundary condition (24) defined out of the operator $\Theta=-\sum_{\ell=2}^{n+1} \partial_{x_{\ell}}^{2}$. Following [Z18, a normalized complete system of generalized eigenfunctions is

$$
\widehat{\psi}_{\xi, k}\left(x_{1}, x\right)=\left[a\left(\xi^{2}+1\right)\right]^{-\frac{1}{2}}\left(\begin{array}{l}
e^{i k x}\left(\cos \left(\xi x_{1}\right)-\xi \sin \left(\xi x_{1}\right)\right) \\
e^{i k x}
\end{array}\right),
$$

where $a=(2 \pi)^{n-1} \frac{\pi}{2}$. A direct computation yields

$$
\begin{aligned}
{\left[\operatorname{pr}_{1} \widehat{\Delta} \widehat{\phi}\right]\left(x_{1}, x\right)=} & \int_{\mathbb{R}_{+} \times \mathbb{R}}\left(k^{2}+\xi^{2}\right) \psi_{\xi, k}\left(x_{1}, x\right)\left[\int_{\mathbb{R}_{+} \times \mathbb{R}} \psi_{\xi, k}\left(y_{1}, y\right) \phi\left(y_{1}, y\right) \mathrm{d} y_{1} \mathrm{~d} y\right] \mathrm{d} \xi \mathrm{d} k \\
{\left[\mathrm{G}_{\Theta} f\right]\left(t, x_{1}, x\right)=} & \int_{\mathbb{R}} \int_{\mathbb{R}_{+} \times \mathbb{R}} \sin \left[\left(\xi^{2}+k^{2}\right)^{\frac{1}{2}}(t-s)\right]\left(\xi^{2}+k^{2}\right)^{-\frac{1}{2}} \psi_{\xi, k}\left(x_{1}, x\right) . \\
& \cdot\left[\int_{\mathbb{R}_{+} \times \mathbb{R}} \psi_{\xi, k}\left(y_{1}, y\right) f\left(s, y_{1}, y\right) \mathrm{d} y_{1} \mathrm{~d} y\right] \mathrm{d} \xi \mathrm{d} k \mathrm{~d} s .
\end{aligned}
$$

\section{Acknowledgements}

The work of C. D. was supported by the University of Pavia. The work of N. D. was supported in part by a research fellowship of the University of Pavia. The work of N. D. and of H. F. was supported in part by a fellowship of the "Progetto Giovani GNFM 2017" under the project "Wave propagation on Lorentzian manifolds with boundaries and applications to algebraic QFT" fostered by the National Group of Mathematical Physics (GNFM-INdAM). We are grateful to Felix Finster, Nadine Grosse, Valter Moretti, Simone Murro and Juan Manuel Pérez-Pardo for the useful comments and discussions. We are grateful to Igor Khavkine for the useful comments, especially concerning Proposition 36.

\section{References}

[AFS18] L. Aké, J. L. Flores, M. Sánchez, "Structure of globally hyperbolic spacetimes with timelike boundary", arXiv:1808.04412 [gr-qc]. 
[AGN16] B. Amman, N. Große and V. Nistor, "Poincaré inequality and well-posedness of the Poisson problem on manifolds with boundary and bounded geometry", arXiv:1611.00281 [math-AP].

[Bac12] A. Bachelot, "New boundary conditions on the time-like conformal infinity of the Anti-de Sitter universe", Comptes Rendus Mathematique, 350 (2012) 359.

[Bar15] C. Bär, "Green-Hyperbolic Operators on Globally Hyperbolic Spacetimes", Comm. Math. Phys. 333 (2015) 1585, arXiv:1310.0738 [math-ph]

[BGP07] C. Bär, N. Ginoux and F. Pfäffle, Wave equation on Lorentzian manifolds and quantization, European Mathematical Society (2007) 194p.

[BEE96] J. K. Beem, P. E. Ehrlich and K. L. Easley, Global Lorentzian geometry, 2nd. ed. CRC Press (1996) 635p.

[Beh10] J. Behrndt "Elliptic boundary value problems with $\lambda$-dependent boundary conditions", J. Diff. Eq. 249 (2010), 2663.

[BL12] J. Behrndt and M. Langer, "Elliptic operators, Dirichlet-to-Neumann maps and quasi boundary triples", in Operator Methods for Boundary Value Problems ed. Hassi, de Snoo and Szafraniec, London Mathematical Society Lecture Notes (2012) Cambridge University Press, 298p.

[BD15] M. Benini and C. Dappiaggi, "Models of free quantum field theories on curved backgrounds," appeared in Chapter 3 of Brunetti et al. (ed.), Advances in algebraic quantum field theory, Math. Phys. Stud. (2015), Springer, arXiv:1505.04298 [math-ph].

[BDH13] M. Benini, C. Dappiaggi and T. P. Hack, "Quantum Field Theory on Curved Backgrounds - A Primer," Int. J. Mod. Phys. A 28 (2013) 1330023 arXiv:1306.0527 [gr-qc]].

[BDS18] M. Benini, C. Dappiaggi and A. Schenkel, "Algebraic quantum field theory on spacetimes with timelike boundary," Annales Henri Poincare 19 (2018) no.8, 2401 [arXiv:1712.06686 [math-ph]].

[CGS09] P. T. Chruściel, G. J. Galloway and D. Solis, "Topological censorship for Kaluza-Klein spacetimes," Annales Henri Poincaré 10 (2009) 893 [arXiv:0808.3233 [gr-qc]].

[DF17] C. Dappiaggi and H. R. C. Ferreira, "On the algebraic quantization of a massive scalar field in anti-de-Sitter spacetime," Rev. Math. Phys. 30 (2017) no.02, 1850004 arXiv:1701.07215 [math-ph]].

[DNP16] C. Dappiaggi, G. Nosari and N. Pinamonti, "The Casimir effect from the point of view of algebraic quantum field theory," Math. Phys. Anal. Geom. 19 (2016) no.2, 12 arXiv:1412.1409 [math$\mathrm{ph}]$.

[DS17] J. Dereziński, D. Siemssen, An Evolution Equation Approach to the Klein-Gordon Operator on Curved Spacetime, arXiv:1709.03911 [math-ph].

[DM91] V. A. Derkach, and M. M. Malamud, "Generalized resolvents and the boundary value problems for Hermitian operators with gaps" (1991) J. Funct. Anal. 95 (1991), 1.

[Eich91] J. Eichhorn, "The Banach manifold structure of the space of metrics on noncompact manifolds," Diff. Geom. Appl. 1 (1991) 89.

[Fel57] W. Feller, Generalized second order differential operators and their lateral conditions, Illinois J. Math. 1 (1957) 459. 
[GGG03] C.G. Gal, G.R. Goldstein and J.A. Goldstein, "Oscillatory boundary conditions for acoustic wave equations", J. Evol. Equ. 3 (2003) 623.

[GM18] N. Große and S. Murro, "The well-posedness of the Cauchy problem for the Dirac operator on globally hyperbolic manifolds with timelike boundary" arXiv:1806.06544 [math.DG]

[GN17] N. Große and V. Nistor, "Neumann and mixed problems on manifolds with boundary and bounded geometry" arXiv:1703.07228 [math-AP].

[GS13] N. Große and C. Schneider, "Sobolev spaces on Riemannian manifolds with bounded geometry: General coordinates and traces" Math. Nachr. 286 (2013) 1586.

[Gru68] G. Grubb, "A characterization of the non-local boundary value problems associated with an elliptic operator", Ann. Sc. Norm. Sup. Pisa (3) 22 (1968) 425.

[Gru70] G. Grubb, Les problèmes aux limites généraux d'un opérateur elliptique provenant de la théorie variationnelle, Bull. Sc. Math., 94, 113 ?157.

[Gru71] G. Grubb, "On coerciveness and semiboundedness of general boundary problems" Israel J. Math. 10 (1971) 32.

[HSF12] S. Hassi, H. De Snoo, F. Szafraniec, (Eds.). (2012). Operator Methods for Boundary Value Problems (London Mathematical Society Lecture Note Series). Cambridge: Cambridge University Press. doi:10.1017/CBO9781139135061

[Heb96] E. Hebey, Sobolev Spaces on Riemannian manifolds, (1996) Springer, 113p.

[Hol12] G. Holzegel "Well-posedness for the massive wave equation on asymptotically anti-de sitter spacetimes", Jour. of Hyp. Diff. Eq., 9 (2012), 239, arXiv:1103.0710 [gr-qc].

[Hör90] L. Hörmander, The Analysis of Linear Partial Differential Operators I 2nd ed. Springer (1990) $438 \mathrm{p}$.

[ILP13] A. Ibort, F. Lledó and J. M. Pérez-Pardo, "Self-adjoint extensions of the Laplace-Beltrami operator and unitaries at the boundary", J. Funct. Anal. 268 (2015) 634.

[ILP14] A. Ibort, F. Lledó and J. M. Pérez-Pardo, "On Self-Adjoint Extensions and Symmetries in Quantum Mechanics," Annales Henri Poincare 16 (2015) no.10, 2367.

[Lee00] J.M. Lee Introduction to Smooth Manifolds, 2nd ed. (2013) Springer 706p.

[LM72] J.L. Lions and E. Magenes, Nonhomogeneous boundary value problems and applications I. Grundlehren der mathematischen Wissenschaften, Springer, 1972.

[Mal92] M. Malamud, "On a formula for the generalized resolvents of a non-densely defined Hermitian operator," Ukr. Math. J. 44 (1992), 1522.

[Mor18] V. Moretti, Spectral Theory and Quantum Mechanics, 2nd ed. (2018) Springer, 950p.

[Pos18] A. Posilicano, Self-adjoint Extensions of Restrictions, Operators and Matrices 2 (2008) 483, arXiv:math-ph/0703078.

[ReSi81] M. Reed, B. Simon, Methods of modern mathematical physics I: Functional Analysis, (1981) Academic Press, 400p. 
[San06] M. Sanchez "On causality and closed geodesics of compact Lorentzian manifolds and static spacetimes" Diff. Geom. Appl. 24 (2006) 21.

[Sch01] T. Schick, "Manifolds with boundary and of bounded geometry", Math. Nachr. 233 (2001) 103.

[Sol06] D. A. Solis, Global properties of asymptotically de Sitter and anti de Sitter spacetimes, $\mathrm{PhD}$ thesis, University of Miami (2006), arXiv:1803.01171 [gr-qc]

[Tri92] H. Triebel, Theory of Function Spaces II, (1992) Birkhauser, 370p.

[Ue73] T. Ueno, "Wave equation with Wentzell's boundary condition and a related semigroup on the boundary, I." Proc. Japan Acad. 49 (1973), 672.

[Vas12] A. Vasy "The wave equation on asymptotically anti de sitter spaces", Analysis and PDE, 5 (2012) 81.

[Wro17] M. Wrochna, "The holographic Hadamard condition on asymptotically Anti-de Sitter spacetimes," Lett. Math. Phys. 107 (2017) 2291, arXiv:1612.01203 [math-ph].

[Z18] J. Zahn, "Generalized Wentzell Boundary Conditions and Quantum Field Theory", Ann. Henri Poinc. 19 (2018) 163. 